\title{
Pacific
}

Journal of

Mathematics

\section{INVARIANTS OF TOTALLY REAL LEFSCHETZ FIBRATIONS}

NERMin SALEPCi 


\title{
INVARIANTS OF TOTALLY REAL LEFSCHETZ FIBRATIONS
}

\author{
NERMIN SALEPCi
}

\begin{abstract}
We introduce certain invariants of real Lefschetz fibrations and call these invariants real Lefschetz chains. We prove that if the fiber genus is greater than 1, then the real Lefschetz chains are complete invariants of totally real Lefschetz fibrations. If however the fiber genus is 1 , real Lefschetz chains are not sufficient to distinguish real Lefschetz fibrations. We show that by adding a certain binary decoration to real Lefschetz chains, we get a complete invariant.
\end{abstract}

\section{Introduction}

This note is devoted to a topological study of Lefschetz fibrations equipped with certain $\mathbb{Z}_{2}$ actions compatible with the fiber structure. The action is generated by an involution, which is called a real structure. Intuitively, real structures are topological generalizations of the complex conjugation on complex algebraic varieties defined over the reals. Real Lefschetz fibrations appear, for instance, as blow-ups of pencils of hyperplane sections of complex projective algebraic surfaces defined by real polynomial equations. Regular fibers of real Lefschetz fibrations are compact oriented smooth genus $g$ surfaces, while singular fibers have a single node. The invariant fibers, called the real fibers, inherit a real structure from the real structure of the total space. We focus on fibrations whose critical values are all fixed by the action and call such fibrations totally real. We also assume that the fixed point set of the base space is oriented. We use the term directed to indicate such fibrations.

The main results of this article are exhibited in Sections 6 and 8 in which we treat the cases of fiber genus $g>1$ and $g=1$, respectively. In Section 6, we introduce real Lefschetz chains and prove that if $g>1$, real Lefschetz chains are complete invariants of directed genus $g$ totally real Lefschetz fibrations over the disk (Corollary 6.4). The case of $g=1$ (elliptic fibrations) is considered in Theorem 8.1. We show that directed totally real elliptic Lefschetz fibrations over $D^{2}$ are determined uniquely by their decorated real Lefschetz chains. In both cases we study extensions of such fibrations to fibrations over a sphere and obtain complete invariants of directed totally real Lefschetz fibrations over a sphere.

MSC2010: primary 55R15, 55R55; secondary 57M05.

Keywords: Lefschetz fibrations, real structure, monodromy. 
It is possible to give a purely combinatorial shape to decorated real Lefschetz chains. We discuss such combinatorial objects, which we call necklace diagrams, and their applications in [Salepci 2012]; see [Degtyarev 2011; Degtyarev and Salepci 2011] for other applications of necklace diagrams.

This paper is organized as follows. In Section 2, we settle the definitions and introduce basic notions. Section 3 is devoted to the topological classification of equivariant neighborhoods of real singular fibers. We show that real Lefschetz fibrations around real singular fibers are determined by the pair consisting of the inherited real structure on one of the nearby regular real fibers and the vanishing cycle that is invariant under the action of the real structure. We call such a pair a real code.

Real Lefschetz chains are, indeed, sequences of real codes each of which is associated to a neighborhood of a real singular fiber. Obviously, each real Lefschetz fibration with real critical values defines a real Lefschetz chain that is, by definition, invariant of the fibration. The natural question to ask is to what extent real Lefschetz chains determine the fibration.

In Section 4, we compute the fundamental group of the components of the space of real structures on a genus $g$ surface. These computations are applied in Section 5 where we define a strong boundary fiber sum (that is, the boundary fiber sum of $\mathbb{C}$ marked real Lefschetz fibrations) and show that if the fiber genus is greater than 1 , then the strong boundary fiber sum is well defined. Section 6 is devoted to $\mathbb{C}$ marked genus $g>1$ fibrations. We show that directed $\mathbb{C}$-marked genus $g>1$ totally real Lefschetz fibrations are classified by their strong real Lefschetz chains. As a corollary, we obtain the result for nonmarked fibrations.

Because of the different geometric nature of the surfaces of genus $g>1$ and $g=1$, we apply slightly different techniques to deal with the case of $g=1$. In Section 7, we define a boundary fiber sum of nonmarked real elliptic Lefschetz fibrations. We observe that the boundary fiber sum is not always well defined. This observation leads to a decoration of directed totally real Lefschetz chains. In the last section, we introduce decorated real Lefschetz chains and prove that they are complete invariants of real elliptic Lefschetz fibrations. We also study extensions of such fibrations to fibrations over a sphere.

\section{Basic definitions}

Throughout the paper $X$ will stand for a compact connected oriented smooth 4manifold and $B$ for a compact connected oriented smooth 2-manifold.

Definition 2.1. A real structure $c_{X}$ on a smooth 4-manifold $X$ is an orientationpreserving involution $c_{X}^{2}=\mathrm{id}$, such that the set Fix $\left(c_{X}\right)$ of fixed points of $c_{X}$ is empty or of the middle dimension. 
Two real structures $c_{X}$ and $c_{X}^{\prime}$ are considered equivalent if there is an orientationpreserving diffeomorphism $\psi: X \rightarrow X$ such that $\psi \circ c_{X}=c_{X}^{\prime} \circ \psi$.

A real structure $c_{B}$ on a smooth 2-manifold $B$ is an orientation-reversing involution $B \rightarrow B$. Such structures are similarly considered up to conjugation by orientation-preserving diffeomorphisms of $B$.

This definition mimics the properties of the standard complex conjugation on complex manifolds. Actually, around a fixed point, every real structure defined as above behaves like complex conjugation.

We will call a manifold together with a real structure a real manifold and the fixed point set the real part.

Remark 2.2. It is well known that for given $g$ there is a finite number of equivalence classes of real structures on a genus $g$ surface $\Sigma_{g}$. These classes can be distinguished by their types and the number of real components. Namely, one distinguishes two types of real structures: separating and nonseparating. A real structure is called separating if the complement of its real part has two connected components; otherwise we call it nonseparating (indeed, in the first case the quotient surface $\Sigma_{g} / c$ is orientable while in the second case it is not). The number of real components of a real structure (note that the real part forms the boundary of $\left.\Sigma_{g} / c\right)$, can be at most $g+1$. This estimate is known as Harnack inequality. By looking at the possible number of connected components of the real part, one can see that on $\Sigma_{g}$ there are $1+[g / 2]$ separating real structures and $g+1$ nonseparating ones. A significant property of the case of genus 1 surfaces is that the number of real components, which can be 0,1 or 2 , is enough to distinguish the real structures.

In this article we stick to the following definition of Lefschetz fibrations.

Definition 2.3. A Lefschetz fibration is a surjective smooth map $\pi: X \rightarrow B$ such that

- $\pi(\partial X)=\partial B$ and the restriction $\partial X \rightarrow \partial B$ of $\pi$ is a submersion;

- $\pi$ has only a finite number of critical points (that is, the points where $d \pi$ is degenerate), all the critical points belong to $X \backslash \partial X$, and their images are distinct points of $B \backslash \partial B$; and

- around each of the critical points one can choose orientation-preserving charts $\psi: U \rightarrow \mathbb{C}^{2}$ and $\phi: V \rightarrow \mathbb{C}$ so that $\phi \circ \pi \circ \psi^{-1}$ is given by $\left(z_{1}, z_{2}\right) \rightarrow z_{1}^{2}+z_{2}^{2}$.

When we want to specify the genus of the nonsingular fibers, we prefer calling them genus $g$ Lefschetz fibrations. In particular, we will use the term elliptic Lefschetz fibrations when the genus is equal to one. For each integer $g$, we will fix a closed oriented surface of genus $g$, which will serve as a model for the fibers, and denote it by $\Sigma_{g}$. In what follows we will always assume that a Lefschetz fibration is relatively minimal; that, is none of its fibers contains a self intersection -1 sphere. 
Definition 2.4. A real structure on a Lefschetz fibration $\pi: X \rightarrow B$ is a pair of real structures $\left(c_{X}, c_{B}\right)$ of $X$ and $B$ such that the diagram

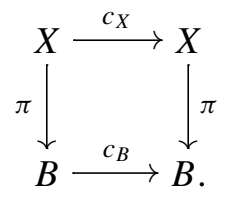

commutes. A Lefschetz fibration equipped with a real structure is called a real Lefschetz fibration and is sometimes referred as RLF. When the fiber genus is 1, we call it a real elliptic Lefschetz fibration (abbreviated RELF).

Definition 2.5. An $\mathbb{R}$-marked $\mathrm{RLF}$ is a triple $(\pi, b, \rho)$ consisting of a real Lefschetz fibration $\pi: X \rightarrow B$, a real regular value $b$ and a diffeomorphism $\rho: \Sigma_{g} \rightarrow F_{b}$ such that $\left.c_{X}\right|_{F_{b}} \circ \rho=\rho \circ c$, where $c: \Sigma_{g} \rightarrow \Sigma_{g}$ is a real structure. Note that if $\partial B \neq \varnothing$, then $b$ will be chosen in $\partial B$.

A $\mathbb{C}$-marked RLF is a triple $(\pi,\{m, \bar{m}\},\{\rho, \bar{\rho}\})$, including a real Lefschetz fibration $\pi: X \rightarrow B$, a pair of regular values $m, \bar{m}=c_{B}(m)$ and a pair of diffeomorphisms $\rho: \Sigma_{g} \rightarrow F_{m}$ and $\bar{\rho}=\left.c_{X}\right|_{F_{m}} \circ \rho: \Sigma_{g} \rightarrow F_{\bar{m}}$, where $F_{m}$ and $F_{\bar{m}}=c_{X}\left(F_{m}\right)$ are the fibers over $m$ and $\bar{m}$, respectively. As in the case of $\mathbb{R}$-marking, if $\partial B \neq \varnothing$, then we choose $m$ in $\partial B$. When precision is not needed we will denote $F_{m}$ and $F_{\bar{m}}$ by $F$ and $\bar{F}$, respectively.

Two real Lefschetz fibrations $\pi: X \rightarrow B$ and $\pi^{\prime}: X^{\prime} \rightarrow B^{\prime}$ are said to be isomorphic if there exist orientation-preserving diffeomorphisms $H: X \rightarrow X^{\prime}$ and $h: B \rightarrow B^{\prime}$ such that this diagram is commutative:

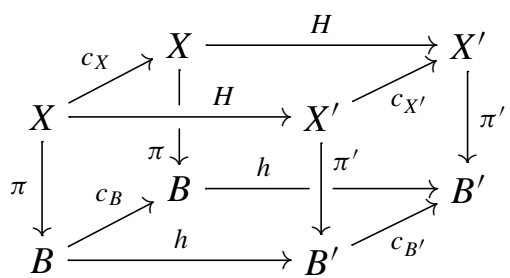

Two $\mathbb{R}$-marked RLFs are called isomorphic if they are isomorphic as RLFs such that $h(b)=b^{\prime}$ and the following diagram is commutative:

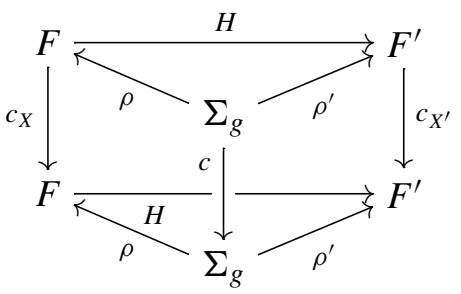


Two $\mathbb{C}$-marked RLFs are called isomorphic if they are isomorphic as RLFs and the following diagram is well defined and commutative:

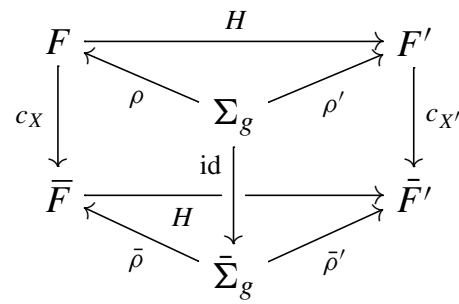

Definition 2.6. A real Lefschetz fibration $\pi: X \rightarrow B$ is called directed if the real part of $\left(B, c_{B}\right)$ is oriented. (If $c_{B}$ is separating, then we consider an orientation on the real part inherited from one of the halves $B \backslash \operatorname{Fix}\left(c_{B}\right)$.)

Two directed RLFs are isomorphic if they are isomorphic as RLFs with the additional condition that the diffeomorphism $h: B \rightarrow B$ preserves the chosen orientation on the real part.

Unless otherwise stated, all fibrations we consider are directed.

Remark 2.7. The notion of Lefschetz fibration can be slightly generalized to cover the case of fibrations whose fibers have nonempty boundary. Then, $X$ turns into a manifold with corners and its boundary $\partial X$ becomes naturally divided into two parts, the vertical boundary $\partial^{v} X$ that is the inverse image $\pi^{-1}(\partial B)$, and the horizontal boundary $\partial^{h} X$ that is formed by the boundaries of the fibers. We call such fibrations Lefschetz fibrations with boundary.

\section{Elementary real Lefschetz fibrations}

In this section, we classify real structures on a neighborhood of a real singular fiber of a real Lefschetz fibration. Such a neighborhood can be viewed as a Lefschetz fibration over a disc $D^{2}$ with a unique critical value $q=0 \in D^{2}$. We call such a fibration an elementary real Lefschetz fibration. Without loss of generality, we may assume that the real structure on $D^{2}$ is the standard one, conj, induced from $\mathbb{C} \supset D^{2}$.

Let $\pi: X \rightarrow D^{2}$ be an elementary RLF. By definition, there exist equivariant local charts $\left(U, \phi_{U}\right)$ and $\left(V, \phi_{V}\right)$ around the critical point $p \in \pi^{-1}(0)$ and the critical value $0 \in D^{2}$, respectively, such that $U$ and $V$ are closed discs and $\left.\pi\right|_{U}:\left(U, c_{U}\right) \rightarrow\left(V\right.$, conj) is equivariantly isomorphic (via $\phi_{U}$ and $\left.\phi_{V}\right)$ to either of $\xi_{ \pm}:\left(E_{ \pm}\right.$, conj $) \rightarrow\left(D_{\epsilon}\right.$, conj $)$, where

$$
E_{ \pm}=\left\{\left(z_{1}, z_{2}\right) \in \mathbb{C}^{2}:\left|z_{1}\right| \leq \sqrt{\epsilon},\left|z_{1}^{2} \pm z_{2}^{2}\right| \leq \epsilon^{2}\right\}
$$

and

$$
D_{\epsilon}=\left\{t \in \mathbb{C}:|t| \leq \epsilon^{2}\right\} \quad \text { for } 0<\epsilon<1 \text {, }
$$



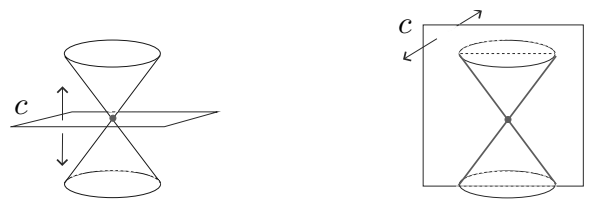

$$
z_{1}^{2}+z_{2}^{2}=0
$$$$
z_{1}^{2}-z_{2}^{2}=0
$$

real part

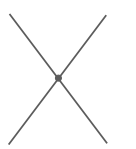

Figure 1. Actions of real structures on the singular fibers of $\xi_{ \pm}$.

with $\xi_{ \pm}\left(z_{1}, z_{2}\right)=z_{1}^{2} \pm z_{2}^{2}$

The real local models above, $\xi_{ \pm}: E_{ \pm} \rightarrow D_{\epsilon}$, can be seen as two real structures on the neighborhood of a critical point. These two real structures are not equivalent. The difference can be seen already at the level of the singular fibers: In the case of $\xi_{+}$, the two branches are imaginary and they are interchanged by the complex conjugation; in the case of $\xi_{-}$the two branches are both real (see Figure 1).

To understand the action of the real structures on the regular real fibers of $\xi_{ \pm}$, we can use the branched covering defined by the projection $\left(z_{1}, z_{2}\right) \rightarrow z_{1}$. Thus:

- In the case of $\xi_{+}$, there are two types of real regular fibers; the fibers $F_{t}$ with $t<0$ have no real points, their vanishing cycles have invariant representatives (that is, $c\left(a_{t}\right)=a_{t}$ set-theoretically), and in this case, $c$ acts on the invariant vanishing cycles as an antipodal involution; the fibers $F_{t}$ with $t>0$ have a circle as their real part and this circle is an invariant (pointwise fixed) representative of the vanishing cycle.

- In the case of $\xi_{-}$, all the real regular fibers are of the same type and the real part of such a fiber consists of two arcs each having its endpoints on the two different boundary components of the fiber; the vanishing cycles have invariant representatives, and $c$ acts on them as a reflection.

Using the ramified covering $\left(z_{1}, z_{2}\right) \rightarrow z_{1}$, we observe that the horizontal boundary of the fibration $\xi_{ \pm}$is equivariantly trivial and has a distinguished equivariant trivialization. Moreover, since the complement of $U$ in $\pi^{-1}(V)$ does not contain any critical point, $X$ can be written as union of two RLFs with boundary: One of them, $U \rightarrow V$, is isomorphic to $\xi_{ \pm}: E_{ \pm} \rightarrow D_{\epsilon}$, and the other one is isomorphic to the trivial real fiber bundle $R \rightarrow D_{\epsilon}$ whose real fibers are equivariantly diffeomorphic to the complement of an open regular neighborhood of the vanishing cycle $a \subset F_{b}$. The action of the complex conjugation on the boundary components of the real fibers of $R \rightarrow D_{\epsilon}$ determines the type $\xi_{ \pm}: E_{ \pm} \rightarrow D_{\epsilon}$ of the model glued to $R \rightarrow D_{\epsilon}$ : In the case of $\xi_{+}$, it switches the boundary components while in the 

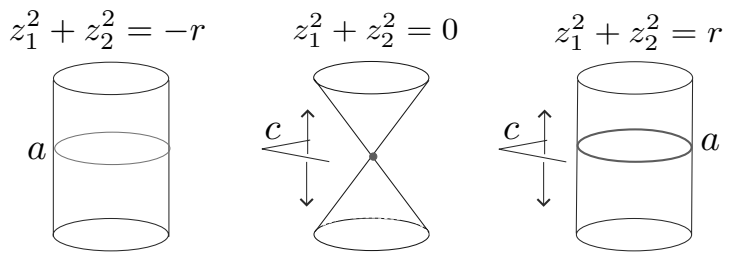

real part

$\begin{array}{ccc}-\boldsymbol{\bullet} & \stackrel{0}{\bullet} \\ & \left(z_{1}, z_{2}\right) & \rightarrow z_{1}^{2}+z_{2}^{2}\end{array}$
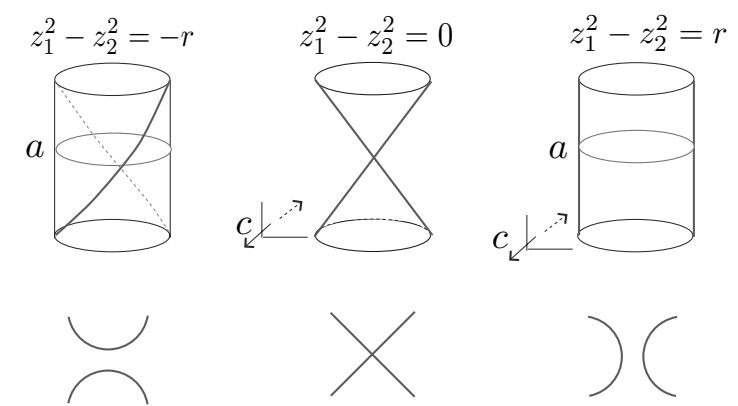

real part

$\begin{array}{cc}-? & \stackrel{0}{\cdot} \quad\left(z_{1}, z_{2}\right) \\ & \rightarrow z_{1}^{2}-z_{2}^{2}\end{array}$

Figure 2. Nearby regular fibers of $\xi_{ \pm}$and the vanishing cycles.

case of $\xi_{-}$, boundary components are preserved (and the complex conjugation acts as a reflection on each of them).

We use the decomposition above to get first a classification of directed $\mathbb{R}$-marked elementary RLF, and then discuss the cases of $\mathbb{C}$-marked and nonmarked fibrations.

Let $\mathscr{A}_{g}^{c}$ denote the set of equivariant isotopy classes of noncontractible curves on a real surface $\left(\Sigma_{g}, c\right)$, let $\mathscr{V}_{g}^{c}$ denote the set of equivariant isotopy classes of noncontractible embeddings $v: S^{1} \times I \rightarrow \Sigma_{g}$ such that $c \circ v=v$, and let $\mathscr{L}_{g}^{\mathbb{R}, c}$ denote the set of isomorphism classes of directed $\mathbb{R}$-marked elementary real Lefschetz fibrations whose distinguished fiber is identified with $\left(\Sigma_{g}, c\right)$.

We consider the map $\Omega: \mathscr{V}_{g}^{c} \rightarrow \mathscr{L}_{g}^{\mathbb{R}, c}$ defined as follows. Let $[v]$ be a class in $\mathscr{V}_{g}^{c}$ with a representative $v$. As $c \circ v=v$, the closure $\Sigma_{g}^{v}$ of $\Sigma_{g} \backslash v\left(S^{1} \times I\right)$ inherits a real structure from $\left(\Sigma_{g}, c\right)$. Let $R^{v}=\Sigma_{g}^{v} \times D_{\epsilon} \rightarrow D_{\epsilon}$ be the trivial real fibration with the real structure $c_{R^{v}}=(c$, conj $): R^{v} \rightarrow R^{v}$ and let $E_{ \pm}^{v} \rightarrow D_{\epsilon}$ denote the model $\xi_{ \pm}: E \rightarrow D_{\epsilon}$ whose marked fiber is identified with $v\left(S^{1} \times I\right)$. Depending on the real structure on the horizontal boundary $S^{1} \times D_{\epsilon} \rightarrow D_{\epsilon}$ (where the real structure 
on $S^{1} \times D_{\epsilon}$ is taken as $\left(c_{\partial \Sigma_{g}^{v}}\right.$, conj)) of $R^{v} \rightarrow D_{\epsilon}$, we choose either of $E_{ \pm}^{v} \rightarrow D_{\epsilon}$. We then glue $R^{v} \rightarrow D_{\epsilon}$ and the suitable model $E_{ \pm}^{v} \rightarrow D_{\epsilon}$ along their horizontal trivial boundaries to get a fibration in $\mathscr{L}_{g}^{\mathbb{R}, c}$.

Lemma 3.1. $\Omega: \mathscr{V}_{g}^{c} \rightarrow \mathscr{L}_{g}^{\mathbb{R}, c}$ is well defined.

Proof. Let $v_{t}: S^{1} \times I \rightarrow \Sigma_{g}$ be an isotopy between $v_{0}$ and $v_{1}$. Then, there exists an equivariant ambient isotopy $\Psi_{t}: \Sigma_{g} \rightarrow \Sigma_{g}$ such that $\Psi_{0}=\mathrm{id}$ and $v_{t}=\Psi_{t} \circ v_{0}$ with $\Psi_{t} \circ c=c \circ \Psi_{t}$ for all $t$. The diffeomorphism $\Psi_{1}$ induces equivariant diffeomorphisms $\Psi_{1}^{R}: R^{\nu_{0}} \rightarrow R^{\nu_{1}}$ and $\Psi_{1}^{E}: E_{ \pm}^{\nu_{0}} \rightarrow E_{ \pm}^{\nu_{1}}$ that respect the fibrations and the gluing; thus, it gives an isomorphism of the images $\Omega\left(\left[v_{0}\right]\right)$ and $\Omega\left(\left[v_{1}\right]\right)$ as $\mathbb{R}$-marked fibrations.

Since $c \circ v=v$, we have $\left.c\left(v\left(S^{1} \times\left\{\frac{1}{2}\right\}\right)\right)=v\left(S^{1} \times\left\{\frac{1}{2}\right\}\right)\right)$. Hence, we can define $\varepsilon: \mathscr{V}_{g}^{c} \rightarrow \mathscr{A}_{g}^{c}$ such that $\varepsilon([v])=\left[v\left(S^{1} \times\left\{\frac{1}{2}\right\}\right)\right]$. This mapping is two-to-one. Since the monodromy does not depend on the orientation of the vanishing cycle, there exists a well-defined mapping $\hat{\Omega}$ such that the following diagram commutes:

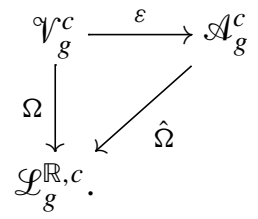

Theorem 3.2. $\hat{\Omega}: \mathscr{A}_{g}^{c} \rightarrow \mathscr{L}_{g}^{\mathbb{R}, c}$ is a bijection.

Proof. As discussed in the beginning of the section, any elementary RLF can be divided equivariantly into two RLFs with boundary: an equivariant neighborhood of the critical point (isomorphic to one of the models, $\xi_{ \pm}$) and the complement of this neighborhood (isomorphic to a trivial real Lefschetz fibration). Such a decomposition defines the equivariant isotopy class of the vanishing cycle. Thus, $\hat{\Omega}$ is surjective.

To show that $\hat{\Omega}$ is injective, let us consider the classes $[a],\left[a^{\prime}\right] \in \mathscr{A}_{g}^{c}$ such that $\hat{\Omega}([a])=\hat{\Omega}\left(\left[a^{\prime}\right]\right)$. Let $\pi: X \rightarrow D_{\epsilon}$ and $\pi^{\prime}: X^{\prime} \rightarrow D_{\epsilon}$ denote the images of $[a]$ and $\left[a^{\prime}\right]$, respectively. Since $\hat{\Omega}$ is well defined, there exist equivariant orientationpreserving diffeomorphisms $H: X \rightarrow X^{\prime}$ and $h: D_{\epsilon} \rightarrow D_{\epsilon}$ such that we have the commutative diagrams
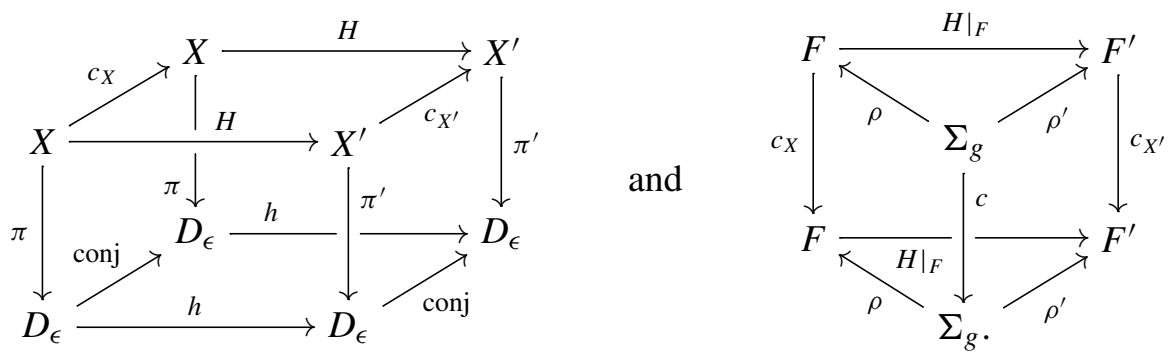


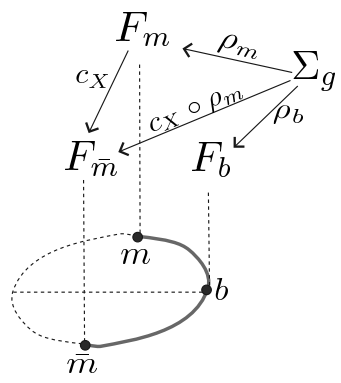

Figure 3. Relation between $\mathbb{R}$-marking and $\mathbb{C}$-marking.

Clearly, $H(\rho(a))$ is equivariantly isotopic to $\rho^{\prime}\left(a^{\prime}\right)$, where $a$ and $a^{\prime}$ are representatives of $[a]$ and $\left[a^{\prime}\right]$, respectively. Moreover, since $\left.H\right|_{F} \circ \rho=\rho^{\prime}$, we have $H(\rho(a))=\rho^{\prime}(a)$, so $\rho^{\prime}(a)$ is equivariant isotopic to $\rho^{\prime}\left(a^{\prime}\right)$.

For $t \in[0,1]$, let $\psi_{t}: F^{\prime} \rightarrow F^{\prime}$ such that $\psi_{0}=\mathrm{id}$ and $\psi_{1}\left(\rho^{\prime}(a)\right)=\rho^{\prime}\left(a^{\prime}\right)$ and that $\psi_{t} \circ c^{\prime}=c^{\prime} \circ \psi_{t}$. Then, $\Psi_{t}=\rho^{\prime-1} \circ \psi_{t} \circ \rho^{\prime}: \Sigma_{g} \rightarrow \Sigma_{g}$ is the required isotopy between $a$ and $a^{\prime}$.

Theorem 3.2 shows that $c$-equivariant isotopy classes of vanishing cycles on $\left(\Sigma_{g}, c\right)$ classify directed $\mathbb{R}$-marked elementary RLFs. To obtain a classification for directed $\mathbb{C}$-marked RLFs, we study the difference between two $\mathbb{C}$-markings; see Figure 3.

Let $\left(\{m, \bar{m}\},\left\{\rho_{m}, c_{X} \circ \rho_{m}\right\}\right)$ be a $\mathbb{C}$-marking on a directed RLF $\pi: X \rightarrow D^{2}$. The complement, $\partial D^{2} \backslash\{m, \bar{m}\}$, has two pieces $S_{ \pm}$(left/right semicircles) distinguished by the direction. By considering a trivialization of the fibration over the piece of $S_{+}$connecting $m$ to the marked real point $b$ (the trivialization over the piece connecting $\bar{m}$ to the real point obtain by the symmetry), we can pull the marking $\rho_{m}: \Sigma_{g} \rightarrow F_{m}$ to $F_{b}$ in order to obtain a marking $\rho_{b}: \Sigma_{g} \rightarrow F_{b}$ and a real structure $c=\rho_{b}^{-1} \circ c_{X} \circ \rho_{b}: \Sigma_{g} \rightarrow \Sigma_{g}$. Any other trivialization results in another marking isotopic to $\rho_{b}$ and a real structure isotopic to $c: \Sigma_{g} \rightarrow \Sigma_{g}$. Hence, a directed elementary $\mathbb{C}$-marked RLF defines a vanishing cycle defined up to $c$-equivariant isotopy, where the real structure $c$ is also considered up to isotopy.

Definition 3.3. A pair $(c, a)$ of a real structure $c: \Sigma_{g} \rightarrow \Sigma_{g}$ and a noncontractible simple closed curve $a \in \Sigma_{g}$ is called a real code if $c(a)=a$.

Two real codes $\left(c_{0}, a_{0}\right)$ and $\left(c_{1}, a_{1}\right)$ are said to be isotopic if there exist a pair $\left(c_{t}, a_{t}\right)$ of isotopies of real structures and vanishing cycles such that $c_{t}\left(a_{t}\right)=a_{t}$ for all $t \in[0,1]$. Two real codes $\left(c_{0}, a_{0}\right)$ and $\left(c_{1}, a_{1}\right)$ are called conjugate if there is an orientation-preserving diffeomorphism $\phi: \Sigma_{g} \rightarrow \Sigma_{g}$ such that $\phi \circ c_{0}=c_{1} \circ \phi$ and that $\phi\left(a_{0}\right)$ is isotopic to $a_{1}$.

We denote the isotopy class of the real code by $[c, a]$ and the conjugacy class by $\{c, a\}$. 
Proposition 3.4. There is a bijection between the isomorphism classes of directed $\mathbb{C}$-marked elementary RLFs and the isotopy classes of real codes.

Proof. We already discussed how to assign a real code to a directed $\mathbb{C}$-marked elementary RLF. It is straightforward to check that this map is well defined and surjective.

To show that it is injective, we consider two isotopy classes $\left[c_{1}, a_{1}\right]$ and $\left[c_{2}, a_{2}\right]$ such that $\left[c_{1}, a_{1}\right]=\left[c_{2}, a_{2}\right]$. Let

$\left(\pi_{1}: X_{1} \rightarrow D^{2},\left\{m_{1}, \bar{m}_{1}\right\},\left\{\rho_{m_{1}}, \bar{\rho}_{m_{1}}\right\}\right) \quad$ and $\quad\left(\pi_{2}: X_{2} \rightarrow D^{2},\left\{m_{2}, \bar{m}_{2}\right\},\left\{\rho_{m_{2}}, \bar{\rho}_{m_{2}}\right\}\right)$

be two directed $\mathbb{C}$-marked elementary RLFs associated to the classes $\left[c_{1}, a_{1}\right]$ and $\left[c_{2}, a_{2}\right]$, respectively. We need to show that $\pi_{1}$ and $\pi_{2}$ are isomorphic as directed $\mathbb{C}$-marked RLFs.

Note that we can always choose a representative $c$ for both $\left[c_{1}\right]$ and $\left[c_{2}\right]$ such that $\left[a_{1}\right]=\left[a_{2}\right] \in \mathscr{A}_{g}^{c}$. Then, by Theorem 3.2, $\pi_{1}$ is isomorphic to $\pi_{2}$ as $\mathbb{R}$-marked RLFs. An isomorphism of $\mathbb{R}$-marked RLFs may not preserve the $\mathbb{C}$-markings; however, it can be modified to preserve them.

Up to homotopy one can identify $X_{2}$ with a subset $\stackrel{\circ}{X}_{2}$ of $X_{1}$. Since the difference $X_{1} \backslash \stackrel{\circ}{X}_{2}$ has no singular fiber, one can transform the marking $\stackrel{\circ}{m}_{2}$ of $\stackrel{\circ}{X}_{2}$ to $m_{1}$, preserving the real marking and the trivializations over the corresponding paths, $S_{+}$and $\dot{S}_{+}$(see Figure 4 ). This way we get an isomorphism of $\mathbb{C}$-marked RLFs preserving the isomorphism class of $\mathbb{R}$-marked RLFs.

For fibrations without marking we allow $[c, a]$ to change by an equivariant diffeomorphism. Hence, we have the following:

Corollary 3.5. There is a bijection between the set of conjugacy classes of real codes and the set of classes of directed nonmarked elementary real Lefschetz fibrations.

Remark 3.6. As the classification of real structures on a genus $g$ surface is known, it is possible to enumerate the conjugacy classes $\{c, a\}$ of real codes. In the case when $a$ is nonseparating, there are 6 classes if $g=1 ; 8 g-3$ classes if $g>1$ and

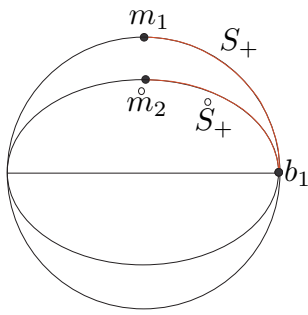

Figure 4. The difference of two $\mathbb{C}$-markings. 
is odd; and $8 g-4$ classes otherwise. The formulas for separating curves can be found in [Salepci 2007].

Remark 3.7. There is no preferable real fiber over the boundary of the disk if the fibration is not directed. Thus, to an elementary nondirected RLF, we can associate two real codes $\left(c_{-}, a_{-}\right)$and $\left(c_{+}, a_{+}\right)$extracted from the "left" and "right" real fibers, respectively. It is a fundamental property of the monodromies of real Lefschetz fibrations that the real structures $c_{-}$and $c_{+}$are related by the monodromy such that $c_{+} \circ c_{-}=t_{a_{-}}=t_{a_{+}}$; see [Salepci 2010].

\section{Equivariant diffeomorphisms and the space of real structures}

In this section we compute the fundamental group of the space of real structures on a genus $g$ surface. The computations will be essential in next sections.

Let $\mathscr{C}^{c}\left(\Sigma_{g}\right)$ denote the space of real structures on $\Sigma_{g}$ that are isotopic to a fixed real structure $c$, and let $\operatorname{Diff}_{0}\left(\Sigma_{g}\right)$ denote the group of orientation-preserving diffeomorphisms of $\Sigma_{g}$ that are isotopic to the identity. We consider two subgroups of $\operatorname{Diff}_{0}\left(\Sigma_{g}\right)$ : One, denoted $\operatorname{Diff}_{0}^{c}\left(\Sigma_{g}\right)$, consists of those diffeomorphisms that commute with $c$, and the other, $\operatorname{Diff}_{0}\left(\Sigma_{g}, c\right)$, is the group of diffeomorphisms that are $c$-equivariantly isotopic to the identity. The group $\operatorname{Diff}_{0}\left(\Sigma_{g}\right)$ acts transitively on $\mathscr{C}^{c}\left(\Sigma_{g}\right)$ by conjugation. The stabilizer of this action is the group $\operatorname{Diff}_{0}^{c}\left(\Sigma_{g}\right)$. Hence, $\mathscr{C}^{c}\left(\Sigma_{g}\right)$ can be identified with the homogeneous space $\operatorname{Diff}_{0}\left(\Sigma_{g}\right) / \operatorname{Diff}_{0}^{c}\left(\Sigma_{g}\right)$.

Lemma 4.1. The space $\operatorname{Diff}_{0}^{c}\left(\Sigma_{g}\right)$ is connected for all $c: \Sigma_{g} \rightarrow \Sigma_{g}$ if $g>1$, and for $c: \Sigma_{g} \rightarrow \Sigma_{g}$, which has one real component, if $g=1$.

Proof. We will use different techniques for the cases $g>1$ and $g=1$.

The case of $g>1$ : We consider the fiber bundle description of conformal structures on $\Sigma_{g}$, introduced in [Earle and Eells 1969]. Let $\operatorname{Conf}_{\Sigma_{g}}$ denote the space of conformal structures on $\Sigma_{g}$ equipped with $C^{\infty}$-topology. The group $\operatorname{Diff}_{0}\left(\Sigma_{g}\right)$ acts on Conf $\Sigma_{\Sigma_{g}}$ by composition from the right. This action is proper, continuous, and effective; hence, $\operatorname{Conf}_{\Sigma_{g}} \rightarrow \operatorname{Conf}_{\Sigma_{g}} / \operatorname{Diff}_{0}\left(\Sigma_{g}\right)$ is a principal $\operatorname{Diff}_{0}\left(\Sigma_{g}\right)$-fiber bundle; see [Earle and Eells 1969]. The quotient is the Teichmüller space of $\Sigma_{g}$, denoted Teich $\Sigma_{\Sigma_{g}}$. Note that conformal structures can be seen as equivalence classes of Riemannian metrics with respect to the relation that two Riemannian metrics are equivalent if they differ by a positive function on $\Sigma_{g}$. Let Riem $\Sigma_{g}$ denote the space of Riemannian metrics on $\Sigma_{g}$. Then, we have the fibrations

$$
\begin{aligned}
&\left\{u: \Sigma_{g} \rightarrow \mathbb{R}: u>0\right\} \longrightarrow \operatorname{Riem}_{\Sigma_{g}} \\
& p_{2} \downarrow \operatorname{Conf}_{\Sigma_{g}} \\
& p_{1} \downarrow \\
& \operatorname{Tiff}_{0}\left(\Sigma_{g}\right) \longrightarrow \text { Teich }_{\Sigma_{g}}
\end{aligned}
$$


The real structure $c$ has an action $\operatorname{Diff}_{0}\left(\Sigma_{g}\right)$ by conjugation. This action extends to $\operatorname{Conf}_{\Sigma_{g}}$ and Riem $\Sigma_{\Sigma_{g}}$ as follows: Fix a section $s: \operatorname{Teich}_{\Sigma_{g}} \rightarrow \operatorname{Conf}_{\Sigma_{g}}$ of the bundle $p_{1}$ and consider a family of diffeomorphisms $\phi_{\zeta}^{s}: \operatorname{Diff}_{0}\left(\Sigma_{g}\right) \rightarrow p_{1}^{-1}(\zeta)$ parametrized by Teich $\Sigma_{\Sigma_{g}}$ such that $\phi_{\zeta}^{s}(\mathrm{id})=s(\zeta)$. Let $s(\zeta)=\left[\mu_{x}\right]$ for some Riemannian metric $\mu_{x}$ on $\Sigma_{g}$. Then, define $\phi_{\zeta}^{s}(f(x))=\left[\mu_{f(x)}\right]$ for all $f \in \operatorname{Diff}_{0}\left(\Sigma_{g}\right)$. The action of the real structure, thus, can be written as $c .\left[\mu_{f(x)}\right]=\left[\mu_{c \circ f \circ c(x)}\right]$. Clearly the definition does not depend on the choice of the representative of the class $\left[\mu_{f(x)}\right]$, so the action extends to $\operatorname{Riem}_{\Sigma_{g}}$.

Let $\operatorname{Fix}_{\operatorname{Conf}_{\Sigma_{g}}}(c)$ denote the set of fixed points of the action of $c$ on $\operatorname{Conf}_{\Sigma_{g}}$ and let $\operatorname{Fix}_{\operatorname{Riem}_{\Sigma_{g}}}(c)$ be the set of fixed points on $\operatorname{Riem}_{\Sigma_{g}}$. Note that $s(\zeta)=\phi_{\zeta}^{s}$ (id) is in $\operatorname{Fix}_{\operatorname{Conf}_{\Sigma_{g}}}(c)$ for all $\zeta \in \operatorname{Teich}_{\Sigma_{g}}$. Indeed, each $\left[\mu_{f(x)}\right]$ for $f \in \operatorname{Diff}_{0}^{c}\left(\Sigma_{1}\right)$ is in $\operatorname{Fix}_{\operatorname{Conf}_{\Sigma_{g}}}(c)$.

The space Fix $\operatorname{Conf}_{\Sigma g}(c)$ is connected. If $\operatorname{Fix}_{\operatorname{Conf}_{\Sigma_{g}}}(c)$ were disconnected, then the inverse image Fix $_{\operatorname{Riem}_{\Sigma_{g}}}(c)$ would also be disconnected in Riem $\Sigma_{g}$. However, it is known that Riem $\Sigma_{\Sigma_{g}}$ is convex; thus, $\operatorname{Fix}_{\operatorname{Riem}_{\Sigma_{g}}}(c)$ is convex, so it is connected. Therefore, $\operatorname{Fix}_{\operatorname{Conf}_{\Sigma_{g}}}(c) \cap \operatorname{Diff}_{0}\left(\Sigma_{g}\right)=\operatorname{Diff}_{0}^{c}\left(\Sigma_{g}\right)$ is connected since $\operatorname{Fix}_{\operatorname{Conf}_{\Sigma_{g}}}(c)$ is a union of sections.

The case of $g=1$ : If $c$ has one real component, then the quotient $\Sigma_{1} / c$ is the Möbius band. The space of diffeomorphisms of the Möbius band has two connected components [Hamstrom 1965]: the identity component and the component of the diffeomorphism induced from the reflection of $I \times I$ with respect to $I \times \frac{1}{2}$ (if the Möbius band is obtained from $I \times I$, we identify the points $t \times 0$ with the points $1-t \times 1$ for $t \in I=[0,1])$. This diffeomorphism is not isotopic to the identity because before identifying the ends it reverses the orientation of $I \times I$, and it lifts to a diffeomorphism of $\Sigma_{1}$ (considered as the obvious quotient of $[-1,1] \times[-1,1]$ ) induced from the central symmetry of $[-1,1] \times[-1,1]$. This diffeomorphism is not isotopic to the identity on $\Sigma_{1}$ since it reverses the orientation of the real curve.

Therefore, we have

$$
\left\{f: \Sigma_{1} / c \rightarrow \Sigma_{1} / c: \hat{f}: \Sigma_{1} \rightarrow \Sigma_{1} \text { is isotopic to id }\right\}=\left\{f: \Sigma_{1} / c \rightarrow \Sigma_{1} / c: f \cong \mathrm{id}\right\} .
$$

The former is identified by $\operatorname{Diff}_{0}^{c}\left(\Sigma_{1}\right)$ and the latter is connected.

Lemma 4.2. For any real structure $c: \Sigma_{g} \rightarrow \Sigma_{g}$,

$$
\pi_{1}\left(\operatorname{Diff}_{0}\left(\Sigma_{g}\right) / \operatorname{Diff}_{0}\left(\Sigma_{g}, c\right), \text { id }\right)= \begin{cases}0 & \text { if } g>1, \\ \mathbb{Z} & \text { if } g=1 .\end{cases}
$$

Proof. Note that the subgroup $\operatorname{Diff}_{0}\left(\Sigma_{g}, c\right)$ acts on $\operatorname{Diff}_{0}\left(\Sigma_{g}\right)$ by composition from the left. Such an action is free, so $\operatorname{Diff}_{0}\left(\Sigma_{g}\right) \rightarrow \operatorname{Diff}_{0}\left(\Sigma_{g}\right) / \operatorname{Diff}_{0}\left(\Sigma_{g}, c\right)$ is a $\operatorname{Diff}_{0}\left(\Sigma_{g}, c\right)$-fiber bundle. The fibers, $\operatorname{Diff}_{0}\left(\Sigma_{g}, c\right)$, can be identified with the group $\operatorname{Diff}_{0}\left(\Sigma_{g} / c\right)$ because the lifting of diffeomorphisms of $\Sigma_{g} / c$ can always be assured by means of the orientation double cover of $\Sigma_{g} / c$. (Note that if $c$ is nonseparating, 
then $\Sigma_{g} / c$ is nonorientable. In this case, $\operatorname{Diff}_{0}\left(\Sigma_{g} / c\right)$ denotes the space of all diffeomorphisms of $\Sigma_{g} / c$ and $\operatorname{Diff}_{0}\left(\Sigma_{g} / c\right)$ is component of the identity.)

Now, we consider the long exact homotopy sequence of this fibration:

$$
\begin{aligned}
\cdots \rightarrow & \pi_{2}\left(\operatorname{Diff}_{0}\left(\Sigma_{g}\right)\right) \rightarrow \pi_{2}\left(\operatorname{Diff}_{0}\left(\Sigma_{g}\right) / \operatorname{Diff}_{0}\left(\Sigma_{g}, c\right)\right) \rightarrow \pi_{1}\left(\operatorname{Diff}_{0}\left(\Sigma_{g}, c\right)\right) \\
& \rightarrow \pi_{1}\left(\operatorname{Diff}_{0}\left(\Sigma_{g}\right)\right) \rightarrow \pi_{1}\left(\operatorname{Diff}_{0}\left(\Sigma_{g}\right) / \operatorname{Diff}_{0}\left(\Sigma_{g}, c\right)\right) \rightarrow \pi_{0}\left(\operatorname{Diff}_{0}\left(\Sigma_{g}\right)\right) \rightarrow \cdots .
\end{aligned}
$$

The case of $g>1$ : The space Diff $\left(\Sigma_{g}\right)$ is contractible for $g>1$ [Earle and Eells 1969], as is $\operatorname{Diff}_{0}\left(\Sigma_{g} / c\right)$ [Earle and Schatz 1970]. Therefore, from the homotopy long exact sequence of the fibration we get $\pi_{1}\left(\operatorname{Diff}_{0}\left(\Sigma_{g}\right) / \operatorname{Diff}_{0}\left(\Sigma_{g}, c\right)\right.$, id $)=0$.

The case of $g=1$ : It is known that $\Sigma_{1}$ is a deformation retract of $\operatorname{Diff}_{0}\left(\Sigma_{1}\right)$ [Ivanov 2001], so the space $\operatorname{Diff}_{0}\left(\Sigma_{1}\right)$ can be considered as a group generated by the rotations that lift to the standard translations on the universal cover.

To understand $\operatorname{Diff}_{0}\left(\Sigma_{g}, c\right)$, we first consider the case when $c$ has two real components. Note that, in this case, the quotient $\Sigma_{1} / c$ is topologically an annulus, so $\pi_{1}\left(\operatorname{Diff}_{0}\left(\Sigma_{1} / c\right)\right.$, id $)=\mathbb{Z}$; see [Ivanov 2001]. We fix an identification of $\varrho: \mathbb{C} / \mathbb{Z}^{2} \rightarrow \Sigma_{1}$ such that the real structure $c$ is the one induced from the standard complex conjugation on $\mathbb{C}$. We consider the family

$$
\begin{aligned}
R_{t}^{\prime 1}: \mathbb{C} / \mathbb{Z}^{2} & \rightarrow \mathbb{C} / \mathbb{Z}^{2}, & R_{t}^{\prime 2}: \mathbb{C} / \mathbb{Z}^{2} & \rightarrow \mathbb{C} / \mathbb{Z}^{2}, \\
(x+i y)_{\mathbb{Z}^{2}} & \mapsto(x+t+i y)_{\mathbb{Z}^{2}}, & (x+i y)_{\mathbb{Z}^{2}} & \mapsto(x+i(y+t))_{\mathbb{Z}^{2}}
\end{aligned}
$$

of diffeomorphisms, where $t \in[0,1]$ and $(x+i y)_{\mathbb{Z}^{2}}$ denotes the equivalence class of $x+i y$ in $\mathbb{C} / \mathbb{Z}^{2}$. Clearly $R_{0}^{\prime j}=R_{1}^{\prime j}=\mathrm{id}$, and $R_{t}^{\prime j}$ is isotopic to identity for each $t \in[0,1]$ and $j=1,2$. The homotopy classes of $R_{t}^{1}=\varrho \circ R_{t}^{\prime 1} \circ \varrho^{-1}$ and $R_{t}^{2}=\varrho \circ R_{t}^{\prime 2} \circ \varrho^{-1}$ form a basis of $\pi_{1}\left(\operatorname{Diff}_{0}\left(\Sigma_{1}\right)\right.$, id). Moreover, with respect to the identification $\varrho$, each diffeomorphism $R_{t}^{1}$ is in $\operatorname{Diff}_{0}\left(\Sigma_{1}, c\right)$, so the loop $R_{t}^{1}$ is a generator of $\pi_{1}\left(\operatorname{Diff}_{0}\left(\Sigma_{1}, c\right)\right.$, id). Thus, from the homotopy exact sequence we get $\pi_{1}\left(\operatorname{Diff}_{0}\left(\Sigma_{1}\right) / \operatorname{Diff}_{0}\left(\Sigma_{1}, c\right)\right.$, id $)=\mathbb{Z}$.

If $c$ has no real component, then the quotient $\Sigma_{1} / c$ is a Klein bottle, so the group $\operatorname{Diff}_{0}\left(\Sigma_{1} / c\right)$ is isomorphic to $S^{1}$ and is generated by the rotation that lifts to a translation in the universal cover of the Klein bottle [Hamstrom 1965]. Let us now fix an identification $\varrho: \mathbb{R}^{2} / \mathbb{Z}^{2} \rightarrow \Sigma_{1}$ such that the real structure $c$ is induced from the real structure

$$
\mathbb{R}^{2} / \mathbb{Z}^{2} \rightarrow \mathbb{R} / \mathbb{Z}^{2}, \quad(x, y)_{\mathbb{Z}^{2}} \mapsto\left(x+\frac{1}{2},-y\right)_{\mathbb{Z}^{2}} .
$$

The classes of family of diffeomorphisms $R_{t}^{j}=\varrho \circ R^{\prime j} \circ \varrho^{-1}$ for $j=1,2$, where

$$
\begin{aligned}
R_{t}^{\prime 1}: \mathbb{R}^{2} / \mathbb{Z}^{2} & \rightarrow \mathbb{R}^{2} / \mathbb{Z}^{2}, & R_{t}^{\prime 2}: \mathbb{R}^{2} / \mathbb{Z}^{2} \rightarrow \mathbb{R}^{2} / \mathbb{Z}^{2}, \\
(x, y)_{\mathbb{Z}^{2}} & \mapsto(x+t, y)_{\mathbb{Z}^{2}}, & (x, y)_{\mathbb{Z}^{2}} \mapsto(x, y+t)_{\mathbb{Z}^{2}},
\end{aligned}
$$


form a basis of $\pi_{1}\left(\operatorname{Diff}_{0}\left(\Sigma_{1}\right)\right.$, id). With respect to the identification $\varrho$ each diffeomorphism $R_{t}^{1}$ is in $\operatorname{Diff}_{0}\left(\Sigma_{1}, c\right)$, so $R_{t}^{1}$ is a generator of $\pi_{1}\left(\operatorname{Diff}_{0}\left(\Sigma_{1}, c\right)\right.$, id $)=\mathbb{Z}$. Therefore, we get $\pi_{1}\left(\operatorname{Diff}_{0}\left(\Sigma_{1}\right) / \operatorname{Diff}_{0}\left(\Sigma_{1}, c\right)\right.$, id $)=\mathbb{Z}$.

If $c$ has a unique real component, $C$, then the restriction $\left.f\right|_{C}$ of $f \in \operatorname{Diff}_{0}\left(\Sigma_{1}, c\right)$ defines a diffeomorphism of $C$. Such a restriction defines a fibration $\operatorname{Diff}_{0}\left(\Sigma_{1}, c\right) \rightarrow$ $\operatorname{Diff}_{0}(C)$ whose fibers isomorphic to $\operatorname{Diff}_{0}\left(\Sigma_{1}, C\right)=\left\{f \in \operatorname{Diff}_{0}\left(\Sigma_{1}, c\right):\left.f\right|_{C}=\right.$ id $\}$. Note that $\operatorname{Diff}_{0}\left(\Sigma_{1}, C\right) \cong \operatorname{Diff}_{0}\left(\overline{\Sigma_{1} \backslash C}, \partial\right)$ where $\overline{\Sigma_{1} \backslash C}$ denotes the closure of $\Sigma_{1} \backslash C$ and $\operatorname{Diff}_{0}\left(\overline{\Sigma_{1} \backslash C}, \partial\right)$ the group diffeomorphisms of $\overline{\Sigma_{1} \backslash C}$ that are identity on the boundary.

Topologically $\Sigma_{1} \backslash C$ is an annulus, so $\operatorname{Diff}_{0}\left(\overline{\Sigma_{1} \backslash C}, \partial\right)$ is contractible; see [Ivanov 2001]. From the homotopy long exact sequence of the fibration

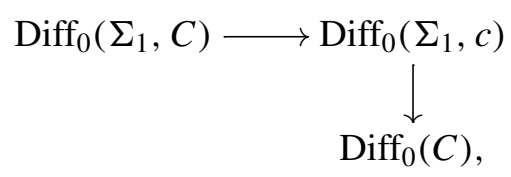

we get $\pi_{k}\left(\operatorname{Diff}_{0}\left(\Sigma_{1}, c\right)\right.$, id $) \cong \pi_{k}\left(\operatorname{Diff}_{0}(C)\right.$, id $)$ for all $k$.

Let us now choose an identification $\varrho: \mathbb{C} / \Lambda \rightarrow \Sigma_{1}$, where $\Lambda$ is the lattice generated by $v_{1}=(1 / \sqrt{2}, 1 / \sqrt{2})$ and $v_{2}=(1 / \sqrt{2},-1 / \sqrt{2})$. Then, the real structure $c$ can be taken as the one induced from the complex conjugation on $\mathbb{C}$.

We consider $R_{i}^{\prime}(t): \mathbb{C} / \Lambda \rightarrow \mathbb{C} / \Lambda, t \in[0,1]$ such that

$$
\begin{aligned}
& {R^{\prime}}_{t}^{1}: \mathbb{C} / \Lambda \quad \rightarrow \mathbb{C} / \Lambda, \quad R_{t}^{\prime 2}: \mathbb{C} / \Lambda \rightarrow \mathbb{C} / \Lambda, \\
& (x+i y)_{\Lambda} \mapsto(x+t+i y)_{\Lambda}, \quad(x+i y)_{\Lambda} \mapsto(x+i(y+t))_{\Lambda} .
\end{aligned}
$$

Again, the classes of $R_{t}^{j}=\varrho \circ R_{t}^{\prime j} \circ \varrho^{-1}$ for $j=1,2$ form a basis for $\operatorname{Diff}_{0}\left(\Sigma_{1}\right)$, while $R_{t}^{1}$ can be taken as a generator for $\pi_{1}\left(\operatorname{Diff}_{0}\left(\Sigma_{1}, c\right)\right.$, id $)=\mathbb{Z}$. Therefore, $\pi_{1}\left(\operatorname{Diff}_{0}\left(\Sigma_{1}\right) / \operatorname{Diff}_{0}\left(\Sigma_{1}, c\right)\right.$, id $)=\mathbb{Z}$.

Proposition 4.3. For any real structure $c: \Sigma_{g} \rightarrow \Sigma_{g}$,

$$
\pi_{1}\left(\mathscr{C}^{c}\left(\Sigma_{g}\right)\right)=\pi_{1}\left(\operatorname{Diff}_{0}\left(\Sigma_{g}\right) / \operatorname{Diff}_{0}^{c}\left(\Sigma_{g}\right), \text { id }\right)= \begin{cases}0 & \text { if } g>1, \\ \mathbb{Z} & \text { if } g=1 .\end{cases}
$$

Proof. By Lemma 4.1, Diff ${ }_{0}^{c}\left(\Sigma_{g}\right)$ is connected for all real $c: \Sigma_{g} \rightarrow \Sigma_{g}$ with $g>1$ and for the real structure $c: \Sigma_{1} \rightarrow \Sigma_{1}$ that has one real component. Hence, in these cases $\operatorname{Diff}_{0}^{c}\left(\Sigma_{1}\right)=\operatorname{Diff}_{0}\left(\Sigma_{1}, c\right)$, so the result follows from Lemma 4.2.

In the case when $c: \Sigma_{1} \rightarrow \Sigma_{1}$ has 2 real components, the space $\operatorname{Diff}_{0}^{c}\left(\Sigma_{1}\right)$ has two connected components. Note that the diffeomorphism $R_{1 / 2}^{2}$ (induced from the translation $(x+i y)_{\mathbb{Z}^{2}} \rightarrow(x+i(y+1 / 2))_{\mathbb{Z}^{2}}$ on $\left.\mathbb{C} / \mathbb{Z}^{2}\right)$ is equivariant; however, it is not equivariantly isotopic to the identity. Hence, $\operatorname{Diff}_{0}^{c}\left(\Sigma_{1}\right)$ has two components: the component $\operatorname{Diff}_{0}\left(\Sigma_{1}, c\right)$ of the identity and the component of the rotation $R_{1 / 2}^{2}$. (In what follows, we denote $R_{1 / 2}^{2}$ by $R_{1 / 2}$.) 
We identify rotations in $\operatorname{Diff}_{0}\left(\Sigma_{1}\right) \backslash \operatorname{Diff}_{0}\left(\Sigma_{1}, c\right)$ with $S^{1}$ by letting $R_{t}^{2} \rightarrow 2 \pi t$. Then rotations in the quotient $\operatorname{Diff}_{0}\left(\Sigma_{1}\right) / \operatorname{Diff}_{0}^{c}\left(\Sigma_{1}\right)$ are identified with $S^{1} / \theta \sim(\theta+\pi)$, so we have $\pi_{1}\left(\operatorname{Diff}_{0}\left(\Sigma_{1}\right) / \operatorname{Diff}_{0}^{c}\left(\Sigma_{1}\right)\right.$, id $)=\mathbb{Z}$.

The case when $c: \Sigma_{1} \rightarrow \Sigma_{1}$ has no real component can be treated similarly using the identification $\varrho: \mathbb{R}^{2} / \mathbb{Z}^{2} \rightarrow \Sigma_{1}$.

\section{Boundary fiber sum of $\mathbb{C}$-marked real Lefschetz fibrations}

Let $\left(D^{2}\right.$, conj) be a real disk with oriented real part. We denote by $S^{ \pm}$the upper/ lower semicircles of $\partial D^{2}$. We consider also left/right semicircles, denoted by $S_{ \pm}$, and the quarter circles $S_{ \pm}^{ \pm}=S^{ \pm} \cap S_{ \pm}$. (Here directions right/left and up/down are determined by the orientations of $D^{2}$ and its real part.) Let $r_{ \pm}$be the real points of $S_{ \pm}$, and $c_{ \pm}$the real structures on $F_{ \pm}=\pi^{-1}\left(r_{ \pm}\right)$.

Definition 5.1. Let

$$
\left(\pi^{\prime}: X^{\prime} \rightarrow D^{2},\left\{b^{\prime}, \bar{b}^{\prime}\right\},\left\{\rho^{\prime}, \bar{\rho}^{\prime}\right\}\right) \quad \text { and } \quad\left(\pi: X \rightarrow D^{2},\{b, \bar{b}\},\{\rho, \bar{\rho}\}\right)
$$

be two directed $\mathbb{C}$-marked real Lefschetz fibrations such that the real structures $c_{+}^{\prime}$ on $F_{+}^{\prime}$ and $c_{-}$on $F_{-}$induce (via the markings) isotopic real structures on $\Sigma_{g}$. Then, we define the strong boundary fiber sum (the boundary fiber sum of $\mathbb{C}$-marked RLFs) as follows.

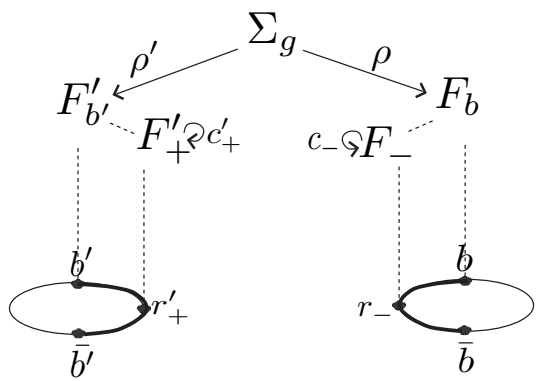

We choose trivializations of $\pi^{\prime-1}\left(S_{+}^{+}\right)$and $\pi^{-1}\left(S_{-}^{+}\right)$such that the pull backs of $c_{+}^{\prime}$ and $c_{-}$give the same real structure $c$ on $\Sigma_{g}$. The trivialization of $\pi^{\prime-1}\left(S_{+}\right)$can be obtained as a union $\Sigma_{g} \times S_{+}^{+} \cup \Sigma_{g} \times S_{+}^{-} /\left(x, 1_{+}\right) \sim\left(c(x), 1_{-}\right)$and similarly $\pi^{-1}\left(S_{-}\right)$ is obtained as $\Sigma_{g} \times S_{-}^{+} \cup \Sigma_{g} \times S_{-}^{-} /\left(x,-1_{+}\right) \sim\left(c(x),-1_{-}\right)$. The strong boundary fiber sum $X^{\prime} \natural_{\Sigma_{g}} X \rightarrow D^{2} \natural D^{2}$ is thus obtained by gluing $\pi^{\prime-1}\left(S_{+}\right)$to $\pi^{-1}\left(S_{-}\right)$via the identity map.

Remark 5.2. (1) In fact, the construction described above creates a manifold with corners, but there is a canonical way to smooth the corners; hence, the strong boundary fiber sum is the manifold obtained by smoothing the corners.

(2) By definition, the strong boundary fiber sum is associative but not commutative. 
(3) The strong boundary fiber sum of $\mathbb{C}$-marked RLFs is naturally $\mathbb{C}$-marked.

Proposition 5.3. If $g>1$, then the strong boundary fiber sum $X^{\prime} \bigsqcup_{\Sigma_{g}} X \rightarrow D^{2}$ of directed $\mathbb{C}$-marked genus $g$ real Lefschetz fibrations is well defined up to isomorphism of $\mathbb{C}$-marked RLFs.

Proof. The boundary fiber sum does not affect the fibrations outside a small neighborhood of the interval where the gluing is made. Let us choose a neighborhood $N$ that is real and far from the critical set. Obviously, the real structures on the fibers over the real points of $N$ are isotopic. Therefore, each fiber sum defines a path in the space of real structures on $\Sigma_{g}$, and the difference of two strong boundary fiber sums gives a loop in this space. Thus, the result follows from the contractibility (shown in Proposition 4.3) of this loop in the case of $g>1$.

\section{Strong real Lefschetz chains associated to $\mathbb{C}$-marked real Lefschetz fibrations}

Let's consider a directed $\mathbb{C}$-marked totally real Lefschetz fibration $\pi: X \rightarrow D^{2}$. We slice $D^{2}$ into smaller discs $D_{1}, D_{2}, \ldots, D_{n}$ (ordered with respect to the orientation of the real part of $\left(D^{2}\right.$, conj)) such that each $D_{i}$ contains only one critical value and the base point $b$ (which is chosen to be the "north pole" as in Figure 5). Let $r_{1}, r_{2}, \ldots, r_{n}, r_{n+1}$ be the real points of $\bigcup_{i=1}^{n} \partial D_{i}$ and let $c_{i}$ be the real structure on $\Sigma_{g}$ pulled back from the inherited real structure of $F_{r_{i}}$.

As claimed in Remark 3.7, we have $c_{i+1} \circ c_{i}=t_{a_{i}}$ for each fibration over $D_{i}$, where $a_{i}$ denotes the corresponding vanishing cycle. As shown in Proposition 3.4, each $\mathbb{C}$-marked real Lefschetz fibration over $D_{i}$ is determined by the isotopy class $\left[c_{i}, a_{i}\right]$ of a real code. Hence, the fibration $\pi: X \rightarrow D^{2}$ yields a sequence of real codes $\left[c_{i}, a_{i}\right]$ satisfying $c_{i+1} \circ c_{i}=t_{a_{i}}$. Clearly this sequence is an invariant of $\pi$.

Definition 6.1. A sequence $\left[c_{1}, a_{1}\right],\left[c_{2}, a_{2}\right], \ldots,\left[c_{n}, a_{n}\right]$ of isotopy classes of real codes is called a strong real Lefschetz chain if we have $c_{i+1} \circ c_{i}=t_{a_{i}}$ for all $i=1, \ldots, n$.

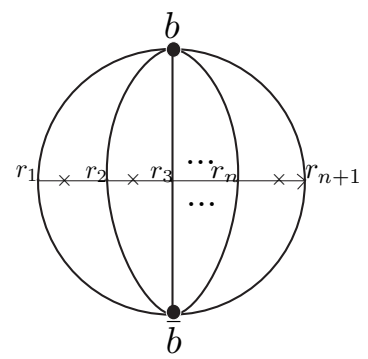

Figure 5. Slicing $D^{2}$ into small discs having one critical value. 
Theorem 6.2. If $g>1$, then there is a one-to-one correspondence between the strong real Lefschetz chains $\left[c_{1}, a_{1}\right],\left[c_{2}, a_{2}\right], \ldots,\left[c_{n}, a_{n}\right]$ and the isomorphism classes of directed $\mathbb{C}$-marked genus $g$ totally real Lefschetz fibrations over $D^{2}$.

Proof. Necessity is clear. As for the converse, we consider the unique class (assured by Proposition 3.4) of directed $\mathbb{C}$-marked elementary real Lefschetz fibration associated to each real code $\left[c_{i}, a_{i}\right]$. We then glue these elementary fibrations (from left to right respecting the order determined by the chain) using the strong boundary fiber sum. The result, thus, follows from Proposition 5.3.

Note that if we consider nonmarked fibrations, then the real codes around real singular fibers are defined up to conjugation. Thus, we are motivated to give the following definition and state the immediate corollary of Theorem 6.2.

Definition 6.3. A sequence $\left\{c_{1}, a_{1}\right\},\left\{c_{2}, a_{2}\right\}, \ldots,\left\{c_{n}, a_{n}\right\}$ of conjugacy classes of real codes is called a real Lefschetz chain if $t_{a_{i}} \circ c_{i}$ is conjugate to $c_{i+1}$ for all $1 \leq i \leq n$.

Corollary 6.4. If $g>1$, then there is a one-to-one correspondence between the real Lefschetz chains $\left\{c_{1}, a_{1}\right\},\left\{c_{2}, a_{2}\right\}, \ldots,\left\{c_{n}, a_{n}\right\}$ and the isomorphism classes of nonmarked directed genus $g$ totally real Lefschetz fibrations over $D^{2}$.

If the total monodromy of the fibration $\pi: X \rightarrow D^{2}$ is the identity, then we can consider the extension of $\pi$ to a fibration $\hat{\pi}: \hat{X} \rightarrow S^{2}$. Two such extensions $\hat{\pi}: \hat{X} \rightarrow S^{2}$ and $\check{\pi}: \check{X} \rightarrow S^{2}$ are considered isomorphic if there is an equivariant orientation-preserving diffeomorphism $H: \hat{X} \rightarrow \check{X}$ such that $\hat{\pi}=\check{\pi} \circ H$.

Proposition 6.5. Let $\pi: X \rightarrow D^{2}$ be a $\mathbb{C}$-marked genus $g$ totally real Lefschetz fibration whose total monodromy is the identity. If $g>1$, then $\pi$ can be extended uniquely up to isomorphism to a totally real Lefschetz fibration over $S^{2}$.

Proof. Once again, the difference of two extensions corresponds to a loop in the space of real structures. Hence, the result follows from Proposition 4.3.

Corollary 6.6. If $g>1$, then there is a one-to-one correspondence between the strong real Lefschetz chains $\left[c_{1}, a_{1}\right],\left[c_{2}, a_{2}\right], \ldots,\left[c_{n}, a_{n}\right]$ such that $c_{n+1} \circ c_{1}=$ $\left(t_{a_{n}} \circ c_{n}\right) \circ c_{1}=\mathrm{id}$ and the isomorphism classes of directed $\mathbb{C}$-marked genus $g$ totally real Lefschetz fibrations over $S^{2}$.

Remark 6.7. It is known that the components of the space of diffeomorphisms of the torus fixing a point are contractible [Earle and Eells 1969], so Theorem 6.2 can be adapted to $\mathbb{C}$-marked real elliptic Lefschetz fibration admitting a real section (a section compatible with the real structures). See [Salepci 2007, Section 5.4] for details. In the next section, we treat the case of nonmarked elliptic Lefschetz fibrations, which possibly do not admit a real section. 


\section{Boundary fiber sum of nonmarked real elliptic Lefschetz fibrations}

To deal with the case of elliptic fibrations, we introduce the boundary fiber sum for nonmarked fibrations. (Although we concentrate on the case of $g(F)=1$, the definition applies to any genus.)

Definition 7.1. Let $\pi^{\prime}: X^{\prime} \rightarrow D^{2}$ and $\pi: X \rightarrow D^{2}$ be two directed nonmarked RLFs. We consider the real fibers $F_{+}^{\prime}$ and $F_{-}$of $\pi^{\prime}$ and $\pi$ over the real points $r_{+}^{\prime}$ and $r_{-}$, respectively. Let us assume that the real structure $c_{+}^{\prime}: F_{+}^{\prime} \rightarrow F_{+}^{\prime}$ is conjugate to $c_{-}: F_{-} \rightarrow F_{-}$. That is, there is an orientation-preserving equivariant diffeomorphism $\phi: F_{+}^{\prime} \rightarrow F_{-}$. Then, the boundary fiber sum of $X^{\prime} \bigsqcup_{F, \phi} X \rightarrow D^{2}$ is obtained by identifying the fibers $F_{+}^{\prime}$ and $F_{-}$via $\phi$, as below.

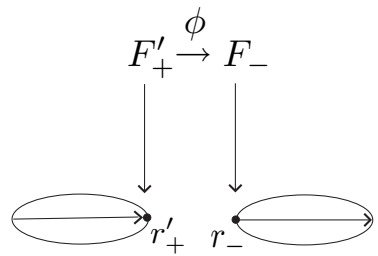

The boundary fiber sum does depend on the choice of $\phi$ in such a way that the two boundary fiber sums defined by the equivariant diffeomorphisms $\phi, \psi$ : $F_{+}^{\prime} \rightarrow F_{-}$are isomorphic, if $\psi \circ \phi^{-1}: F_{-} \rightarrow F_{-}$can be extended to an equivariant diffeomorphism of $X \rightarrow D^{2}$ (or similarly if $\phi^{-1} \circ \psi: F_{+}^{\prime} \rightarrow F_{+}^{\prime}$ can be extended to an equivariant diffeomorphism of $X^{\prime} \rightarrow D^{2}$ ). The necessary and sufficient condition for $\psi \circ \phi^{-1}: F_{-} \rightarrow F_{-}$to extend to an equivariant diffeomorphism of the fibration $X \rightarrow D^{2}$ is that $\psi \circ \phi^{-1}$ takes the unique vanishing cycle $a$ of $X \rightarrow D^{2}$ to a curve equivariantly isotopic to $a$.

Now note that if $c(a)=a$, then $c$ induces an action on $a$. Such an action can be the identity, a reflection, or an antipodal involution. It is not hard to show that if $c: \Sigma_{1} \rightarrow \Sigma_{1}$ has one real component, then $\Sigma_{1}$ contains a unique $c$-equivariant isotopy class of noncontractible curves on which $c$ acts as a reflection, a unique class of curves where the action of $c$ is an antipodal involution, and a unique real curve; if $c$ has 2 real components, then $\Sigma_{1}$ contains no $c$-equivariant isotopy class of curves on which $c$ acts as an antipodal involution, a unique class of curves on which $c$ acts as a reflection, and two classes of real curves (in which case, we call a pair of representatives of different classes $c$-twin curves); if $c$ has no real components, then there exist two $c$-equivariant isotopy classes where $c$ acts as an antipodal involution (as above, a pair of representatives of different classes are called c-twin curves) and no classes of other types. The boundary fiber sum is, therefore, well defined unless the real structure $c$ has no real component or $c$ has two real components one of which is the vanishing cycle $a$. 
Recall that the rotation $R_{1 / 2}$ (introduced in the proof of Proposition 4.3) switches the $c$-twin curves. Hence, $c$-twin curves can be carried to each other via equivariant diffeomorphisms although they are not equivariantly isotopic, so in the case of existence of $c$-twin curves, there is an ambiguity in the definition of the boundary fiber sum $X^{\prime} \natural X \rightarrow D^{2}$ (it can be defined in two ways). To resolve the ambiguity, we should specify how we identify the $c_{+}^{\prime}$-twin curves on the fiber $F_{+}^{\prime}$ in $X^{\prime}$ with the $c_{-}$-twin curves on the fiber $F_{-}$in $X$. In a certain case, namely, if the real structure $c_{+}^{\prime}$ has two real components and acts on the vanishing cycle $a^{\prime}$ as a reflection, the problem of switching $c$-twin curves can be eliminated via the transformation introduced below.

Let $\pi: X \rightarrow D^{2}$ be an elementary directed real elliptic Lefschetz fibration such that the real structure $c_{+}: F_{+} \rightarrow F_{+}$acts on the vanishing cycle as a reflection. As a result, one of $c_{ \pm}: F_{ \pm} \rightarrow F_{ \pm}$has 1 real component while the other has 2 real components. Without loss of generality, we can assume that the real structure $c_{-}$ has 1 real component. Our aim is to construct a transformation $T_{\text {sing }}$ of $X$ that does not change the isomorphism class of the fibration $\pi: X \rightarrow D^{2}$ and that is identity over $S_{-} \subset \partial D^{2}$ and interchanges the real components of $F_{+}$. To construct $T_{\text {sing }}$, we consider the following well known model for elementary elliptic fibrations.

Let $\hat{\Omega}=\left\{z|| \operatorname{Re}(z) \mid \leq \frac{1}{2}, \operatorname{Im}(z) \geq 1\right\} \cup \infty$. This is the subset bounded by $\operatorname{Im}(z) \geq 1$ of the one point compactification of the standard fundamental domain $\left\{z|| \operatorname{Re}(z)\left|\leq \frac{1}{2},\right| z \mid \geq 1\right\}$ of the modular action on $\mathbb{C}$; see Figure 6 .

We consider the real structure $c_{\hat{\Omega}}: \hat{\Omega} \rightarrow \hat{\Omega}$ such that $c_{\hat{\Omega}}(\omega)=\overline{-\omega}$. Let $\Omega$ denote the quotient $\hat{\Omega} / \frac{1}{2}+i y \sim-\frac{1}{2}+i y$. The real structure $c_{\hat{\Omega}}$ induces a real structure on $\Omega$. Note that $\Omega$ is a topological real disc and can be identified with $D^{2}$ so that the real part of $D^{2}$ corresponds to the union of the half-lines $i y$ and $\frac{1}{2}+i y$, where $y \geq 1$. For any $\omega \in \Omega$, the fiber over $\omega$ is given by $F_{\omega}=\mathbb{C} /(\mathbb{Z}+\omega \mathbb{Z})$, where the fiber $F_{\infty}$ has the required nodal-type singularity.
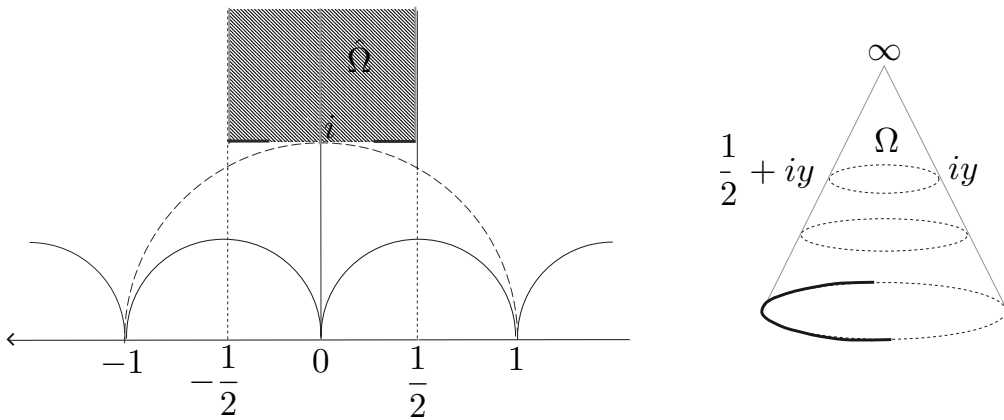

Figure 6. Moduli space of prescribed RELFs. 


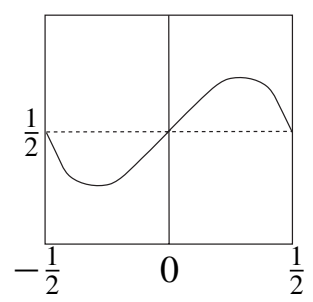

Figure 7. The graph of $f$.

Let $\pi_{\Omega}: X_{\Omega} \rightarrow \Omega$ denote the fibration such that $\pi_{\Omega}^{-1}(\omega)=F_{\omega}=\mathbb{C} /(\mathbb{Z}+\omega \mathbb{Z})$ for all $\omega \in \Omega$. Then, we consider the translation $T_{\Omega}^{\prime}$ defined by

$$
T_{\Omega}^{\prime}: X_{\Omega} \rightarrow X_{\Omega}, \quad(z)_{\mathbb{Z}+\omega \mathbb{Z}} \in F_{\omega} \mapsto(z+\tau(w))_{\mathbb{Z}+\omega \mathbb{Z}} \in F_{\omega},
$$

where $(\cdot)_{\mathbb{Z}+\omega \mathbb{Z}}$ denotes the equivalence class in $\mathbb{C} /(\mathbb{Z}+\omega \mathbb{Z})$.

The map $\tau: \Omega \rightarrow \Omega$ is defined by

$$
\tau(\omega)=-\frac{1}{2}+\left(\frac{1}{2}-f(\operatorname{Re}(\omega))+i\right) \exp (-\operatorname{Im}(\omega)+1),
$$

where $f: \mathbb{R} / \mathbb{Z} \rightarrow \mathbb{R} / \mathbb{Z}$ is a smooth mapping whose graph is as shown in Figure 7 and that satisfies the following properties:

- $f(0)=\frac{1}{2}(\operatorname{modulo} \mathbb{Z})$,

- $f(1-x)=1-f(x),\left(\right.$ which implies $\left.f\left(\frac{1}{2}\right)=\frac{1}{2}\right)$ (modulo $\left.\mathbb{Z}\right)$,

- $f$ is linear on $\left[\frac{1}{4}, \frac{3}{4}\right]$ (modulo $\mathbb{Z}$ ).

Note that $\tau$ has the following properties. (Equations are considered modulo the relation $-\frac{1}{2}+i y \sim \frac{1}{2}+i y$, with $y \geq 1$.)

- $\tau(\overline{-\omega})=\overline{-\tau(\omega)}$.

- $\tau(\infty)=\frac{1}{2}$.

- $\tau\left(\frac{1}{2}+i y\right)=-\frac{1}{2}+i \exp (-y+1)=\frac{1}{2}+i \exp (-y+1)$; in particular, if $y=1$, then $\tau\left(\frac{1}{2}+i\right)=\frac{1}{2}+i$.

- $\tau(i y)=-\frac{1}{2}+i \exp (-y+1)=\frac{1}{2}+i \exp (-y+1)$; in particular, if $y=1$, then $\tau(i)=\frac{1}{2}+i$.

Let $T_{\text {sing }}: X \rightarrow X$ denote the transformation induced from $T_{\text {sing }}^{\prime}: X_{\Omega} \rightarrow X_{\Omega}$. By definition $T_{\text {sing }}$ is equivariant and the identity over $S_{-} \subset \partial D^{2}$, and its restriction to $F_{+}$is the rotation $R_{1 / 2}$. (Figure 8 shows the action of $T_{\text {sing. }}$.)

Lemma 7.2. Let $\pi^{\prime}: X^{\prime} \rightarrow D^{2}$ and $\pi: X \rightarrow D^{2}$ be two nonmarked elementary RELFs such that both $c_{+}^{\prime}$ and $c_{-}$have 2 real components. We assume that the vanishing cycle a of $\pi$ is real with respect to $c_{-}$. Then, the boundary fiber sum $X^{\prime} \natural_{F} X \rightarrow D^{2}$ is well defined if $c_{+}^{\prime}$ acts on the vanishing cycle $a^{\prime}$ as a reflection. 


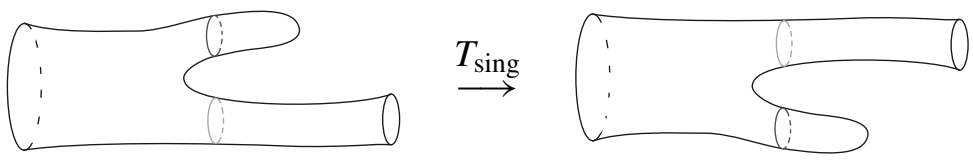

Figure 8. The action of $T_{\text {sing }}$ on the real part (in gray).

Proof. The boundary fiber sums $X^{\prime} \bigsqcup_{F, \phi} X \rightarrow D^{2}$ and $X^{\prime} \bigsqcup_{F, \psi} X \rightarrow D^{2}$ are not isomorphic if $\phi \circ \psi^{-1}(a)$ and $a$ are $c$-twin curves, but in the case when $c_{+}^{\prime}$ acts on the vanishing cycle $a^{\prime}$ as a reflection, we can apply $T_{\text {sing }}$ to $X^{\prime}$ so that $T_{\text {sing }}\left(F_{+}^{\prime}\right)$ differs from the fiber $F_{+}^{\prime}$ by the rotation $R_{1 / 2}$. Hence, $X^{\prime} \natural_{F, \phi} X \rightarrow D^{2}$ is isomorphic to $T_{\text {sing }}\left(X^{\prime}\right) \bigsqcup_{F, \phi \circ R_{1} / 2} X \rightarrow D^{2}$, which is isomorphic to $X^{\prime} \bigsqcup_{F, \psi} X \rightarrow D^{2}$.

\section{Real Lefschetz chains associated to nonmarked real elliptic Lefschetz fibrations}

We now consider a nonmarked directed totally real elliptic Lefschetz fibration $\pi$ : $X \rightarrow D^{2}$, with $q_{1}<q_{2}<\cdots<q_{n}$. Around each critical value $q_{i}$ we choose a small real disc $D_{i}$ such that

$$
D_{i} \cap\left\{q_{1}, q_{2}, \ldots, q_{n}\right\}=\left\{q_{i}\right\} \quad \text { and } \quad D_{i} \cap D_{i+1}=\left\{r_{i+1}\right\} \subset\left[q_{i}, q_{i+1}\right] ;
$$

see Figure 9. Let $c_{i}$ be the real structures on the fibers $F_{r_{i}}$ for $1 \leq i \leq n$ (where $r_{1}$ is the left real point of $\partial D^{2}$ ) and $a_{i}$ be the corresponding vanishing cycle.

By Corollary 3.5, each directed (nonmarked) fibration over $D_{i}$ is classified by the conjugacy class $\left\{c_{i}, a_{i}\right\}$ of the real code. Thus, we can encode the fibration $\pi: X \rightarrow D^{2}$ by the real Lefschetz chain $\left\{c_{1}, a_{1}\right\},\left\{c_{2}, a_{2}\right\}, \ldots,\left\{c_{n}, a_{n}\right\}$.

Clearly, real Lefschetz chains are invariants of directed nonmarked totally real elliptic Lefschetz fibrations over $D^{2}$, but they are not sufficient for classifying such fibrations. Additional information is needed, if for some $i$ the real structure $c_{i}$ has 2 real components and vanishing cycles corresponding to the critical values $q_{i}$ and

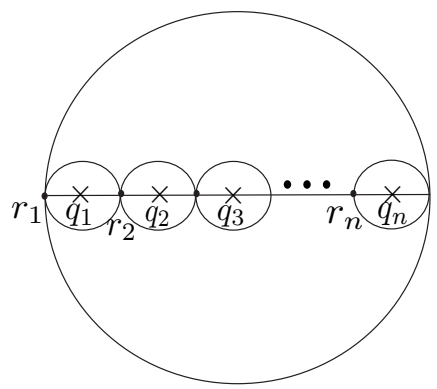

Figure 9. Subdividing $D^{2}$ into smaller discs. 
$\left\{c_{i}, a_{i}\right\}$

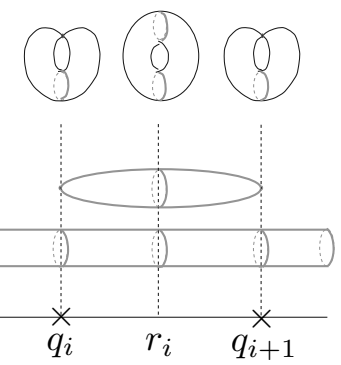

$\left\{c_{i}, a_{i}\right\}$

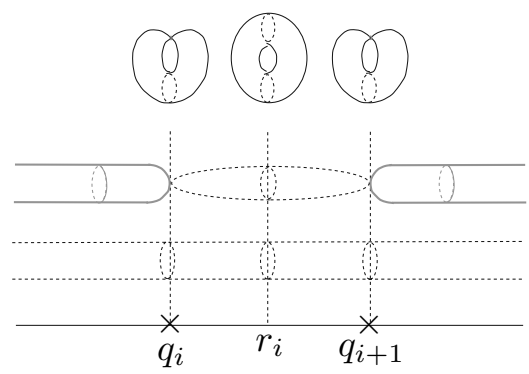

$\left\{c_{i}, a_{i}\right\}^{R}$
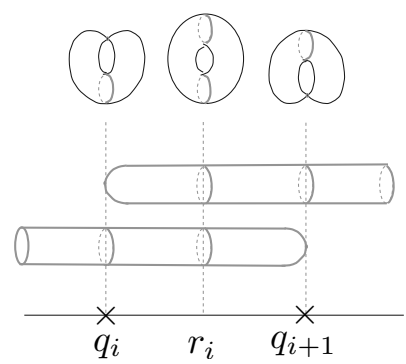

$\left\{c_{i}, a_{i}\right\}^{R}$

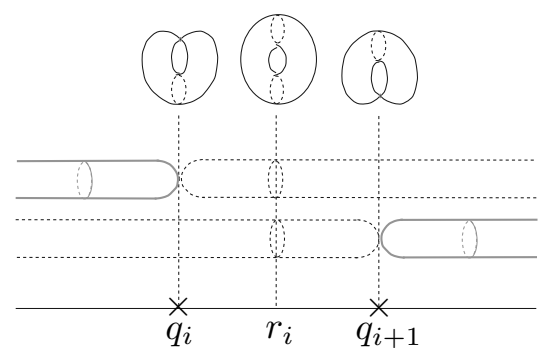

Figure 10. Real parts (in gray) of the fibrations associated to $\left\{c_{i}, a_{i}\right\}$ and $\left\{c_{i}, a_{i}\right\}^{R}$.

$q_{i+1}$ are real or if $c_{i}$ has no real component. Indeed, in these cases the vanishing cycles corresponding to the critical values $q_{i}$ and $q_{i+1}$ can be the same curve, or they can be $c_{i}$-twin curves. If they are $c_{i}$-twin curves, then we mark $\left\{c_{i}, a_{i}\right\}^{R}$ the corresponding real code $\left\{c_{i}, a_{i}\right\}$ by adding $R$ (here $R$ refers to the rotation $R_{1 / 2}$ that interchanges $c$-twin curves). The real Lefschetz chain we obtain is called the decorated real Lefschetz chain. Figure 10 shows all possible configurations of the real locus associated to $\left\{c_{i}, a_{i}\right\}$ and $\left\{c_{i}, a_{i}\right\}^{R}$.

Theorem 8.1. There exists a one-to-one correspondence between the decorated real Lefschetz chains and the isomorphism classes of directed nonmarked totally real elliptic Lefschetz fibrations over $D^{2}$.

Proof. Necessity is clear. As for the converse, we consider the unique class of the directed nonmarked elementary RELF (assured by Corollary 3.5) associated to each real code $\left\{c_{i}, a_{i}\right\}$. Then, we construct the required fibration by gluing elementary fibrations (from left to right) using the boundary fiber sum. As discussed above, the boundary fiber sum is uniquely defined in the case when the real structure on the fiber where the sum is performed has 1 real component or when it has 2 real components and acts on the vanishing cycle of the fibration glued to 


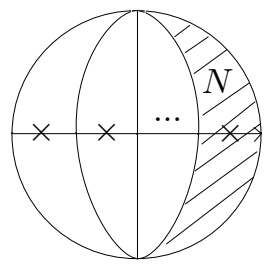

Figure 11. Neighborhood over which $T_{\text {sing }}$ is applied.

right as a reflection. In the case when the real structure has 2 real components and acts as reflection on the vanishing cycle corresponding to the rightmost critical value of the already constructed $\pi^{\prime}: X^{\prime} \rightarrow D^{2}$, the two possible boundary fiber sums are isomorphic by Lemma 7.2 since in this case we can apply $T_{\text {sing }}$ to $X^{\prime}$ (by considering $T_{\text {sing }}$ on a neighborhood $N$ of the last critical value, as shown in Figure 11, and extending it to $X^{\prime}$ as the identity outside of $\pi^{\prime-1}(N)$ ). In all the other cases, the boundary fiber sum is defined uniquely by the decoration.

If $c_{1}$ is conjugate to $c_{n+1}$, then we can consider an extension of $\pi: X \rightarrow D^{2}$ to a fibration over $S^{2}$. As before, in the case when $c_{n+1}$ has no real components or it has 2 real components and both $a_{1}$ and $a_{n}$ are real, a decoration at infinity will be needed.

Proposition 8.2. Let $\pi: X \rightarrow D^{2}$ be a totally real elliptic Lefschetz fibration associated to a decorated real Lefschetz chain. We assume that the real structures $c_{1}$ and $c_{n+1}$ on the fibers over left and respectively right real point of $\partial D^{2}$ are conjugate. If $c_{n+1}$ (and thus $c_{1}$ ) has 1 real component or if $c_{n+1}$ (and thus $c_{1}$ ) has 2 real components and either $c_{n+1}$ acts on the vanishing cycle $a_{n}$ as a reflection, or $c_{1}$ acts on the vanishing cycle $a_{1}$ as a reflection, then $\pi$ extends uniquely to a fibration over $S^{2}$. Otherwise, there are two extensions distinguished by the decoration at infinity.

Proof. An extension of $\pi: X \rightarrow D^{2}$ to a fibration over $S^{2}$ defines a trivialization $\phi: \Sigma_{1} \times S^{1} \rightarrow \pi^{-1}\left(\partial D^{2}\right)$ over the boundary $\partial D^{2}$. Two trivializations $\phi$ and $\phi^{\prime}$ correspond to isomorphic real fibrations if $\phi^{-1} \circ \phi^{\prime}: \Sigma_{1} \times S^{1} \rightarrow \Sigma_{1} \times S^{1}$ can be extended to an equivariant diffeomorphism of $\Sigma_{1} \times D^{2}$ with respect to the real structure $\left(c_{n+1}\right.$, conj) : $\Sigma_{1} \times D^{2} \rightarrow \Sigma_{1} \times D^{2}$. Let $\Phi_{t}=\left(\phi^{-1} \circ \phi^{\prime}\right)_{t}: \Sigma_{1} \rightarrow \Sigma_{1}$, $t \in S^{1}$. Since there is no fixed marking, up to change of marking we assume that $\Phi_{t} \in \operatorname{Diff}_{0}\left(\Sigma_{1}\right)$.

The real structure splits the boundary into two symmetric pieces, so instead of considering an equivariant map over the entire boundary we consider a diffeomorphism over one the symmetric pieces. Let $\Phi_{t}$ for $t \in[0,1]$ denote the family of such diffeomorphisms. This family defines a path in $\operatorname{Diff}_{0}\left(\Sigma_{1}\right)$ whose end points lie in the group $\operatorname{Diff}_{0}^{c_{n+1}}\left(\Sigma_{1}\right)$; therefore, $\Phi_{t}$ defines a relative loop in 


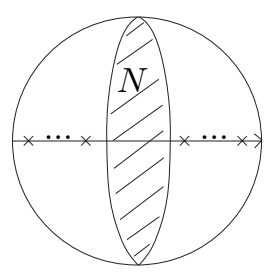

Figure 12. Neighborhood over which $T$ is applied.

$\pi_{1}\left(\operatorname{Diff}_{0}\left(\Sigma_{1}\right), \operatorname{Diff}_{0}^{c_{n+1}}\left(\Sigma_{1}\right)\right)$, and we are interested in the contractibility of this relative loop.

We consider the exact sequence

$$
\begin{aligned}
\cdots \rightarrow \pi_{1}\left(\operatorname{Diff}_{0}^{c_{n+1}}\right) \rightarrow \pi_{1}\left(\text { Diff }_{0}\right) \stackrel{f}{\rightarrow} \pi_{1}\left(\text { Diff }_{0}, \text { Diff }_{0}^{c_{n+1}}\right) & \stackrel{g}{\rightarrow} \pi_{0}\left(\text { Diff }_{0}^{c_{n+1}}\right) \\
\stackrel{h}{\rightarrow} \pi_{0}\left(\text { Diff }_{0}\right) & \rightarrow \pi_{0}\left(\text { Diff }_{0}, \text { Diff }_{0}^{c_{n+1}}\right) \rightarrow 0
\end{aligned}
$$

of the pair $\left(\operatorname{Diff}_{0}\left(\Sigma_{1}\right), \operatorname{Diff}_{0}^{c_{n+1}}\left(\Sigma_{1}\right)\right)$.

In the case when $c_{n+1}$ has one real component, $\operatorname{Diff}_{0}^{c_{n+1}}\left(\Sigma_{1}\right)$ is connected, so the map $h$ is injective; hence $f$ is surjective. Therefore, we can see elements of the group $\pi_{1}\left(\operatorname{Diff}_{0}\left(\Sigma_{1}\right)\right.$, Diff $c_{0}^{c_{n+1}}\left(\Sigma_{1}\right)$, id) as being in $\pi_{1}\left(\operatorname{Diff}_{0}\left(\Sigma_{1}\right)\right.$, id $)$.

In all the other cases, Diff $c_{n+1}^{c_{n+1}}\left(\Sigma_{1}\right)$ has two components. We mark one of the components to make the map $h$ injective when restricted to the marked component. Thus, $g$ becomes the zero map, and so $f$ is surjective over the marked component of Diff ${ }_{0}^{c_{n+1}}\left(\Sigma_{1}\right)$. Note that decoration of real Lefschetz chains distinguishes one of the component of $\operatorname{Diff}_{0}^{c_{n+1}}\left(\Sigma_{1}\right)$; hence, marking one component or other give the two extensions distinguished by the decoration. The distinctive feature of the case when $c_{n+1}$ has 2 real components and acts $a_{n}$ as a reflection (or $c_{1}$ acts on $a_{1}$ as a reflection) is that the transformation $T_{\text {sing }}$ changes one marking to other, so the marking is not essential.

The proposition, thus, follows from Lemma 8.4 in which we show that any relative loop can be made contractible by means of some transformations $T$ of the fibration $\pi: X \rightarrow D^{2}$.

Let us first define the transformation $T$ of real elliptic Lefschetz fibrations that is defined over a regular slice $N$ of $D^{2}$.

Let $\pi: X \rightarrow D^{2}$ be a directed RELF. We consider a real slice $N$ of $D^{2}$ that contains no critical value; see Figure 12.

Let $\xi: I \times I \rightarrow N$, where $I=[0,1]$, be an orientation-preserving diffeomorphism such that first interval corresponds to the real direction on $N$. The fibration over $N$ has no singular fiber; hence, it is trivializable. Let us consider a trivialization 
$\Xi: \Sigma_{1} \times I \times I \rightarrow \pi^{-1}(N)$ such that the following diagram commutes:

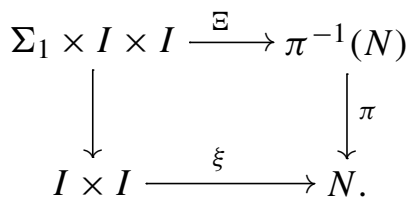

Since $N$ has no critical value, the isotopy type of the real structure on the fibers over the real part of $N$ remains fixed. If the real structure $c$ has 2 real components, then we consider the model $\varrho: \mathbb{C} / \mathbb{Z}^{2} \rightarrow \Sigma_{1}$ and set $\varrho=(\varrho$, id $): \mathbb{C} / \mathbb{Z}^{2} \times I \times I \rightarrow$ $\Sigma_{1} \times I \times I$ to define $T$ as follows:

$$
T^{\prime}: \mathbb{C} / \mathbb{Z}^{2} \times I \times I \rightarrow \mathbb{C} / \mathbb{Z}^{2} \times I \times I, \quad\left((x+i y)_{\mathbb{Z}^{2}}, t, s\right) \mapsto\left((x+t+i y)_{\mathbb{Z}^{2}}, t, s\right) .
$$

Then, we set $T=\Xi \circ\left(\bar{\varrho} \circ T^{\prime} \circ \bar{\varrho}^{-1}\right) \circ \Xi^{-1}$ on $\pi^{-1}(N)$. Since $T$ is the identity at $t=0,1$, we can extend $T$ to $X$ by the identity outside of $\pi^{-1}(N)$.

If $c$ has one real component, we construct $T$ using $\varrho: \mathbb{C} / \Lambda \rightarrow \Sigma_{1}$. Similarly, if $c$ has no real component, then we repeat the same using $\varrho: \mathbb{R}^{2} / \mathbb{Z}^{2} \rightarrow \Sigma_{1}$.

Remark 8.3. First, since the transformation $T$ is defined by a real rotation, $T$ preserves the isomorphism class of the real Lefschetz fibration.

Second, the map $T$ depends only on the isotopy type of $\pi^{-1}(N)$.

Lemma 8.4. Let $\pi: X \rightarrow D^{2}$ be a totally real elliptic Lefschetz fibration. We assume that there exists at least one vanishing cycle on which corresponding real structure acts as a reflection. Then, there exists a generating set for

$$
\pi_{1}\left(\operatorname{Diff}_{0}\left(\Sigma_{1}\right), \text { id }\right)=\mathbb{Z}+\mathbb{Z}
$$

consisting of transformations $T_{ \pm}$for some nonsingular slices $N_{ \pm}$.

Proof. Let $q_{i}$ be the critical value such that the real structure on a nearby regular real fiber acts on the vanishing cycle as a reflection. This assumption assures that the neighboring real fibers have one real component on one side and two real components on the other side of the critical value $q_{i}$. Without loss of generality we can assume that the real structure over a fiber over a real point that lies on the left of $q_{i}$ has two real components. (The other case can be treated similarly.)

We choose an auxiliary $\mathbb{C}$-marking $\left(\{b, \bar{b}\},\left\{\rho: \Sigma_{1} \rightarrow F_{b}, \bar{\rho}: \Sigma_{1} \rightarrow F_{\bar{b}}\right\}\right)$ and fix an identification $\varrho: S^{1} \times S^{1} \rightarrow \Sigma_{1}$. Since the real structure has 2 real components, we can assume that the induced real structure on $S^{1} \times S^{1}$ is the reflection $(\alpha, \beta) \rightarrow(\alpha,-\beta)$. The real part consists of the curves $C_{1}=(\alpha, 0)$ and $C_{2}=(\alpha, \pi)$. Moreover, a representative of the vanishing cycle can be chosen as $(0, \beta)$. As $c_{+}=t_{a_{i}} \circ c_{-}$on $S^{1} \times S^{1}$, the real part of $c_{+}$is the curve $C_{3}$ given homologically by $2 \alpha-\beta$; see Figure 13 . 

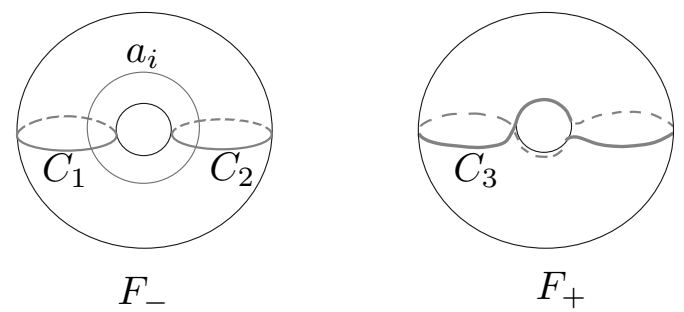

Figure 13. Real fibers (in gray) over the real points neighboring $q_{i}$.

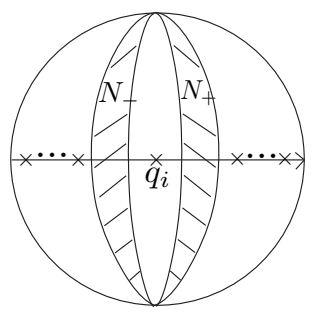

Figure 14. Regular slices $N_{ \pm}$.

We now consider two nonsingular real slices $N_{-}$and $N_{+}$of $D^{2}$ as shown in Figure 14. Let us suppose that the real fibers over $N_{-}$are identified to $F_{-}$while real fibers over $N_{+}$are identified to $F_{+}$(where $F_{ \pm}$are as shown Figure 13). Let $C_{3}^{\prime}$ and $C_{1}^{\prime}$ be curves on $F_{b}$ obtained by pulling back $C_{3} \subset F_{+}$and $C_{1} \subset F_{-}$, respectively. The curves $C_{3}^{\prime}$ and $C_{1}^{\prime}$ intersect at one point, so we can identify $\Sigma_{1}$ with $C_{1}^{\prime} \times C_{3}^{\prime}$ so that rotations along $C_{1}^{\prime}$ and $C_{3}^{\prime}$ generate the group $\operatorname{Diff}_{0}\left(\Sigma_{1}\right.$, id $)$. Hence, $\left\{T_{+}, T_{-}\right\}$generates $\pi_{1}\left(\operatorname{Diff}_{0}\left(\Sigma_{1}\right)\right.$, id $)$.

Theorem 8.1 applies naturally to directed nonmarked RELFs over $D^{2}$ that admit a real section in which a real-case Lefschetz chain does not contain a real code $\left(c_{i}, a_{i}\right)$ where the real structure has no real component. Besides, in the case when the real structure has 2 real components and the vanishing cycle is real, the decoration is not needed since the existence of a real section determines naturally the gluing. Moreover, the extension to a fibration over $S^{2}$ is uniquely defined by the section. Hence we have the following proposition.

Proposition 8.5. Two directed totally real elliptic Lefschetz fibrations over $S^{2}$ admitting a real section and having the same real Lefschetz chain up to cyclic ordering are isomorphic.

Remark 8.6. Indeed, the proposition holds even for fibrations with a fixed real section. If there are only real critical values, then the real sections are determined in a neighborhood of a real part. Moreover, over the real part one can carry one real section to another using the transformations $T$ and double $T_{\text {sing. Indeed, the }}$ 

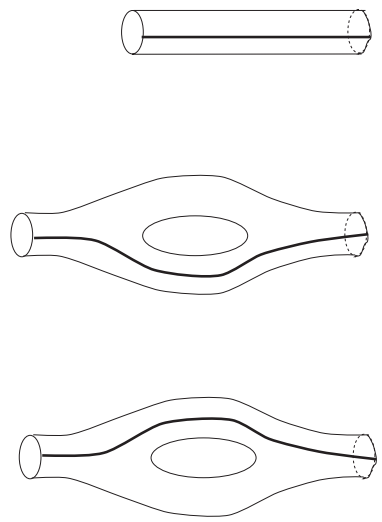
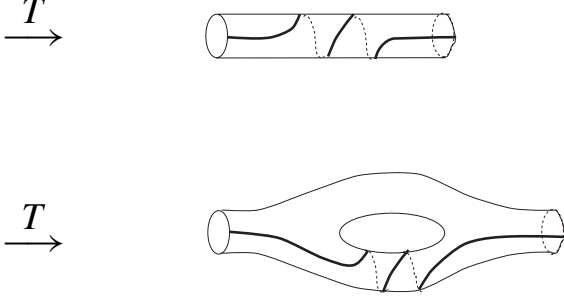

double $T_{\text {sing }}$

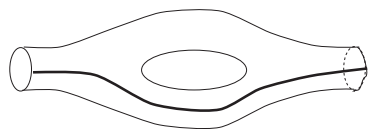

Figure 15. Modification of the real section over the real part.

double $T_{\text {sing }}$ is defined for real Lefschetz fibrations with two critical values where the real structure extracted from the real fiber over a real point between the critical values acts on the vanishing cycles as a reflection. The model we use to define the double $T_{\text {sing }}$ is as follows. Consider the disc $D$ with two critical values as the double cover of a disc with one critical value branched at a regular real point. Let $D_{-}$and $D_{+}$be two corresponding copies of the disk on the branched cover. By pulling back the fibration $X_{\Omega}$ over $D$, we obtain a model fibration over $D_{-} \cup D_{+}$. Thus, we can apply $T_{\text {sing }}$ at the same time to fibrations over $D_{-}$and $D_{+}$. The possible modifications of the section is shown in the Figure 15.

\section{Acknowledgments}

This work is extracted from my thesis. I would like to express my gratitude to my supervisors Sergey Finashin and Viatcheslav Kharlamov for sharing their deep insight and knowledge.

\section{References}

[Degtyarev 2011] A. Degtyarev, "Hurwitz equivalence of braid monodromies and extremal elliptic surfaces", Proc. Lond. Math. Soc. 103:6 (2011), 1083-1120. MR 2861751 Zbl 1234.14030

[Degtyarev and Salepci 2011] A. Degtyarev and N. Salepci, "Products of pairs of Dehn twists and maximal real Lefschetz fibrations", preprint 2011 - 32, Mathematisches Forschungsinstitut Oberwolfach, 2011, available at http://www.mfo.de/scientific-programme/publications/owp/2011/ OWP2011_32.pdf. To appear in Nagoya Math. $J$.

[Earle and Eells 1969] C. J. Earle and J. Eells, "A fibre bundle description of Teichmüller theory", J. Differential Geom. 3:1-2 (1969), 19-43. MR 43 \#2737a Zbl 0185.32901

[Earle and Schatz 1970] C. J. Earle and A. Schatz, "Teichmüller theory for surfaces with boundary", J. Differential Geom. 4:2 (1970), 169-185. MR 43 \#2737b Zbl 0194.52802 
[Hamstrom 1965] M.-E. Hamstrom, "Homotopy properties of the space of homeomorphisms on $P^{2}$ and the Klein bottle", Trans. Amer. Math. Soc. 120:1 (1965), 37-45. MR 32 \#1707 Zbl 0148.17201

[Ivanov 2001] N. V. Ivanov, "Mapping class groups", pp. 523-633 in Handbook of geometric topology, edited by R. J. Daverman and R. B. Sher, Elsevier, Amsterdam, 2001. MR 2003h:57022 Zbl 1002.57001

[Salepci 2007] N. Salepci, Real Lefschetz fibrations, thesis, Université Louis Pasteur, Strasbourg, 2007, available at http://tinyurl.com/Salepci-thesis. MR 2780321 Zbl 1216.55004

[Salepci 2010] N. Salepci, "Real elements in the mapping class group of $T^{2}$ ", Topology Appl. 157:16 (2010), 2580-2590. MR 2011g:57022 Zbl 1213.57024

[Salepci 2012] N. Salepci, "Classification of totally real elliptic Lefschetz fibrations via necklace diagrams”, J. Knot Theory Ramifications 21:9 (2012), 1250089.

Received April 21, 2011.

NERMIN SALEPCi

INSTITUT CAMILLE JORDAN

UNIVERSITÉ LYON I

43, Boulevard du 11 Novembre 1918

69622 VilleurbanNE CEDEX

FRANCE

salepci@math.univ-lyon1.fr

http://math.univ-lyon1.fr/ salepci/ 


\title{
PACIFIC JOURNAL OF MATHEMATICS
}

\author{
http://pacificmath.org \\ Founded in 1951 by \\ E. F. Beckenbach (1906-1982) and F. Wolf (1904-1989)
}

\section{EDITORS}

V. S. Varadarajan (Managing Editor)

Department of Mathematics

University of California

Los Angeles, CA 90095-1555

pacific@math.ucla.edu

Vyjayanthi Chari

Department of Mathematics

University of California

Riverside, CA 92521-0135

chari@math.ucr.edu

\section{Robert Finn}

Department of Mathematics Stanford University

Stanford, CA 94305-2125

finn@math.stanford.edu

Kefeng Liu

Department of Mathematics

University of California

Los Angeles, CA 90095-1555

liu@math.ucla.edu
Darren Long

Department of Mathematics

University of California

Santa Barbara, CA 93106-3080

long@math.ucsb.edu

Jiang-Hua Lu

Department of Mathematics

The University of Hong Kong

Pokfulam Rd., Hong Kong jhlu@maths.hku.hk

Alexander Merkurjev

Department of Mathematics

University of California

Los Angeles, CA 90095-1555

merkurev@math.ucla.edu
Sorin Popa

Department of Mathematics University of California

Los Angeles, CA 90095-1555 popa@math.ucla.edu

Jie Qing

Department of Mathematics

University of California

Santa Cruz, CA 95064

qing@cats.ucsc.edu

Jonathan Rogawski

Department of Mathematics

University of California

Los Angeles, CA 90095-1555

jonr@math.ucla.edu

\section{PRODUCTION}

pacific@math.berkeley.edu

\section{SUPPORTING INSTITUTIONS}

ACADEMIA SINICA, TAIPEI

CALIFORNIA INST. OF TECHNOLOGY INST. DE MATEMÁTICA PURA E APLICADA KEIO UNIVERSITY

MATH. SCIENCES RESEARCH INSTITUTE NEW MEXICO STATE UNIV.

OREGON STATE UNIV.

\author{
STANFORD UNIVERSITY \\ UNIV. OF BRITISH COLUMBIA \\ UNIV. OF CALIFORNIA, BERKELEY \\ UNIV. OF CALIFORNIA, DAVIS \\ UNIV. OF CALIFORNIA, LOS ANGELES \\ UNIV. OF CALIFORNIA, RIVERSIDE \\ UNIV. OF CALIFORNIA, SAN DIEGO \\ UNIV. OF CALIF., SANTA BARBARA
}

\author{
UNIV. OF CALIF., SANTA CRUZ \\ UNIV. OF MONTANA \\ UNIV. OF OREGON \\ UNIV. OF SOUTHERN CALIFORNIA \\ UNIV. OF UTAH \\ UNIV. OF WASHINGTON \\ WASHINGTON STATE UNIVERSITY
}

These supporting institutions contribute to the cost of publication of this Journal, but they are not owners or publishers and have no responsibility for its contents or policies.

See inside back cover or pacificmath.org for submission instructions.

The subscription price for 2012 is US \$420/year for the electronic version, and \$485/year for print and electronic.

Subscriptions, requests for back issues from the last three years and changes of subscribers address should be sent to Pacific Journal of Mathematics, P.O. Box 4163, Berkeley, CA 94704-0163, U.S.A. Prior back issues are obtainable from Periodicals Service Company, 11 Main Street, Germantown, NY 12526-5635. The Pacific Journal of Mathematics is indexed by Mathematical Reviews, Zentralblatt MATH, PASCAL CNRS Index, Referativnyi Zhurnal, Current Mathematical Publications and the Science Citation Index.

The Pacific Journal of Mathematics (ISSN 0030-8730) at the University of California, c/o Department of Mathematics, 969 Evans Hall, Berkeley, CA 94720-3840, is published monthly except July and August. Periodical rate postage paid at Berkeley, CA 94704, and additional mailing offices. POSTMASTER: send address changes to Pacific Journal of Mathematics, P.O. Box 4163, Berkeley, CA 94704-0163.

PJM peer review and production are managed by EditFLOW ${ }^{\mathrm{TM}}$ from Mathematical Sciences Publishers.

PUBLISHED BY PACIFIC JOURNAL OF MATHEMATICS

at the University of California, Berkeley 94720-3840

A NON-PROFIT CORPORATION

Typeset in LATEX

Copyright $(02012$ by Pacific Journal of Mathematics 


\title{
PACIFIC JOURNAL OF MATHEMATICS
}

\author{
Volume $256 \quad$ No. $2 \quad$ April 2012
}

O-operators on associative algebras and associative Yang-Baxter equations

Chengming BAI, Li GuO and Xiang Ni

Botany of irreducible automorphisms of free groups

291

THIERRY COULBOIS and ARNAUd HILION

A note on inverse curvature flows in asymptotically Robertson-Walker 309 spacetimes

Claus GerhardT

Total curvature of graphs after Milnor and Euler

Robert GULLIVER and SUMIO YAMADA

Entire solutions of Donaldson's equation

WEIYONG HE

Energy identity and removable singularities of maps from a Riemann surface with tension field unbounded in $L^{2}$

YONG LUO

Quotients by actions of the derived group of a maximal unipotent subgroup

\section{DMITRI I. PANYUSHEV}

Invariants of totally real Lefschetz fibrations

NERMIN SALEPCI

Stable trace formulas and discrete series multiplicities

STEVEN SPALLONE

Small covers and the Halperin-Carlsson conjecture

\section{YU}

